\title{
A Scalable Multiuser Framework for Video over OFDM Networks: Fairness and Efficiency
}

Guan-Ming Su, Student Member, IEEE, Zhu Han Member, IEEE, Min Wu, Member, IEEE, and K. J. Ray Liu Fellow, IEEE

\begin{abstract}
In this paper, we propose a framework to transmit multiple scalable video programs over downlink multiuser OFDM networks in real time. The framework explores the scalability of the video codec and multi-dimensional diversity of multiuser OFDM systems to achieve the optimal service objectives subject to constraints on delay and limited system resources. We consider two essential service objectives, namely, the fairness and efficiency. Fairness concerns the video quality deviation among users who subscribe the same quality of service, and efficiency relates to how to attain the highest overall video quality using the available system resources. We formulate the fairness problem as minimizing the maximal end-to-end distortion received among all users and the efficiency problem as minimizing total end-to-end distortion of all users. Fast suboptimal algorithms are proposed to solve the above two optimization problems. The simulation results demonstrated that the proposed fairness algorithm outperforms a time division multiple (TDM) algorithm by $0.5 \sim 3 \mathrm{~dB}$ in terms of the worst received video quality among all users. In addition, the proposed framework can achieve a desired tradeoff between fairness and efficiency. For achieving the same average video quality among all users, the proposed framework can provide fairer video quality with $1 \sim 1.8 \mathrm{~dB}$ lower PSNR deviation than a TDM algorithm.
\end{abstract}

\section{Index Terms}

Scalable video coding, multiuser OFDM networks, dynamic resource allocation, multiuser video communications.

Copyright (c) 2006 IEEE. Personal use of this material is permitted. However, permission to use this material for any other purposes must be obtained from the IEEE by sending an email to pubs-permissions@ieee.org.

Manuscript received January 6, 2005; revised December 23, 2005 and July 24, 2006. This work was supported by the U.S. National Science Foundation under Award \#CCR-0133704 and MURI F496200210217. Some preliminary results of this work were presented in the IEEE Global Telecommunications Conference 2004. This paper was recommended by Associate Editor S. Li.

G.-M. Su is with ESS Technology, Fremont, CA 94538 USA \{email: guanming.su@esstech.com\}.

Z. Han is with the Department of Electrical and Computer Engineering, Boise State University, Boise, ID 83725 USA \{email : hanzhu22@gmail.com \}.

M. Wu and K. J. Ray Liu are with the Department of Electrical and Computer Engineering, University of Maryland, College Park, MD 20742 U.S.A \{email: minwu@eng.umd.edu; kjrliu@umd.edu \}

Digital Object Identifier: 


\section{INTRODUCTION}

With the advancement of video compression technology and wide deployment of wireless local area networks (WLAN), transmitting multiple compressed video programs over band-limited wireless fading channel has become an emerging service. A multiuser video transmission system should consider not only the reconstructed video quality of each individual user but also different perspectives from network-level point of view. We consider two essential service objectives, namely, the fairness and efficiency. The first objective regards whether the received video qualities are fair or not for the users who subscribe the same video quality level. The second objective is efficiency, namely, how to achieve the highest overall users' received video qualities with a limited amount of system resources. If the users pay the same price for a certain video quality, the received qualities for these users should be similar. The challenge to attain each objective is how to effectively allocate radio and video resources to each video stream. To facilitate resource management, a system with highly adjustable radio and video resources is preferred. For the radio resource, the wireless communication system should provide high data rates to accommodate multimedia transmission and equip multi-dimensional diversity so that radio resources can be dynamically distributed according to users' needs and channel conditions. For the video source coding, the video codec should have high scalability to aid rate adaptation to achieve the required quality. In this paper, we address the above issues and present a framework to reach a desired tradeoff between fairness and efficiency.

To provide high data transmission rate, orthogonal frequency division multiplex (OFDM) system is a promising modulation scheme and has been adopted in the current technology, such as Digital Audio Broadcasting (DAB), Digital Video Broadcasting (DVB), WLAN standard (IEEE $802.11 \mathrm{a} / \mathrm{g}$ ), and Wireless Metropolitan Area Networks (WMAN) standard (IEEE 802.16a). Compared to the traditional OFDM system, a multiuser OFDM system has higher adjustability for dynamic allocation of resources such as subcarrier, rate, and transmission power. Therefore, a multiuser OFDM system can explore time, frequency, and multiuser diversities to improve system performances, such as throughput [1], [2]. Allocating resources in a multiuser OFDM system is often formulated as an optimization problem. If 
the objective function and system resource constraints are continuous and convex, multiuser iterative waterfilling is an effective solution to maximize system's utility [3]-[5]. However, the multiuser OFDM system often has resources with both continuous and integer valued parameters and systems may also have non-linear or/and non-convex constraints. Thus, obtaining the optimal solution is often $N P$ hard. Through Lagrangian relaxation, an algorithm satisfying users' minimal quality requirement and minimizing the overall transmission power was proposed in [6]. To alleviate the high computational complexity, several suboptimal but computationally efficient algorithms for transmitting generic data were proposed in [7][10]. Unlike generic data, compressed video sources exhibit different characteristics, for example, there is highly bursty rate from frame to frame and different compression complexity from one scene to another scene. Furthermore, a streaming video system has a strict delay constraint that belated video data is useless for its corresponding frame and will cause error propagation for the video frames encoded predictively from this frame. Therefore, the radio resource allocation problem for transmitting video is more difficult than the problem for transmitting generic data. A real-time low-complexity algorithm for transmitting wireless video is desired.

To transmit video programs over wireless networks, a system should be able to adjust the video source bit rates according to the varying channel conditions. A highly scalable video codec is desired since it provides flexibility and convenience in reaching the desired visual quality or the desired bit rate. The Fine Granularity Scalability (FGS) coding and Fine Granular Scalability Temporal (FGST) coding in the MPEG-4 video coding standard can provide high flexibility. However, their overall qualities are worse than the non-scalable coding results, and there remains a non-scalable base layer. The development of 3-D subband video coding [11]-[16] provides an alternative to compress video with full scalability, namely, spatial scalability, temporal scalability, and SNR scalability. Unlike the motion compensated video codec based on block matching (such as H.263 and MPEG-4), the 3-D subband coding explores the spatiotemporal redundancies via a 3-D subband transform. Extending the bit allocation ideas from the EBCOT algorithm for image compression [17], the 3-D embedded wavelet video codec (EWV) 
[16] outperforms MPEG-4 for sequences with low or moderate motion and has comparable performance to MPEG-4 for most high-motion sequences. Moreover, the rate-distortion (R-D) information can be predicted during the encoding procedure and provide a one-to-one mapping between rate and distortion such that we can achieve the desired perceptual quality or the targeted bit rate. Thus, we adopt the EWV codec in the proposed framework as an example. We can easily incorporate other codecs with similar coding strategy into the proposed scheme.

A wireless system transmitting a single video program has been widely studied in the literature [18][20]. To improve the overall system performance, joint source and channel coding has been shown as an effective approach [21]-[27]. When we consider a system transmitting heterogeneous video programs simultaneously, the system has another dimension of diversity to explore since different video scenes have different content complexity: at a given encoded bit rate, some video scenes may have unnecessarily high perceptual quality, while others may have low perceptual quality. It has been shown that joint multiple video source coding can leverage different video content complexities to achieve more desired quality [28]-[32]. Thus, for a multiuser wireless system, the main challenges to achieve the highest system performance are how to allocate limited and shared radio resources to multiple users, how to jointly select video source and radio parameters, and how to deliver video streams to multiple users in real time. A simple solution for a multiuser wireless video system was proposed by assigning subcarriers according to the length of terminal's queue [33]. In this paper, we overcome the aforementioned challenges by allocating resources through a multiuser cross-layer optimization, namely, we formulate the whole system as optimization problems by jointly exploring the diversity of video and radio resources in a cross-layer fashion to optimize the network-level service objectives.

Motivated by the above advantages of multiuser OFDM system and EWV video codec, we propose a framework to provide multiple video streams to different users using dynamic distortion control. The proposed framework has the following features. First, the system dynamically gathers the information of system resources from different components to capture the time-heterogeneity of video sources and 
time-varying characteristics of channel conditions. Subject to delay constraint, the system explores multidimensional diversity among users and across layers, performs joint multiuser cross-layer resource allocation optimization, and then distributes the system resources to each user. The benefit for such joint consideration is the higher utilization of system resources. The simulation results demonstrated that the proposed fairness algorithm outperforms a time division multiple (TDM) algorithm based on traditional WLAN technology by $0.5 \sim 3 \mathrm{~dB}$ in terms of the worst received video quality criterion. Second, extremely fair allocation in such a heterogeneous environment will cause low overall video qualities when some users are trapped in bad channels. On the other hand, optimizing the system efficiency will only cause unfairness among users. To reach the tradeoff between fairness and efficiency, our proposed framework first achieves baseline fairness among all users and then pursuits the high overall system's efficiency. Compared to the TDM algorithm, the proposed framework can provide fairer video quality with $1 \sim 1.8 \mathrm{~dB}$ lower PSNR deviation among all users for achieving the same overall video quality.

This paper is organized as follows. The system architecture for transmitting 3-D EWV over multiuser OFDM networks is described in Section II. In Section III, we concentrate on fairness issue among users and formulate the proposed system as a min-max problem. In Section IV, we focus on system efficiency. The tradeoff between fairness and efficiency and potential solution to increase efficiency through unequal error protection are addressed in Section V. Simulation results are presented in Section VI and conclusions are drawn in Section VII.

\section{SYSTEM DESCRIPTION}

There are three major subsystems in the proposed wireless video system, namely, the video source codec subsystem, the multiuser OFDM subsystem, and the resource allocator subsystem. We first review the video source codec subsystem along with the corresponding R-D characteristics, and describe the multiuser OFDM subsystem with adaptive modulation and adaptive channel coding. Then, we present the proposed framework for transmitting multiple scalable video bitstreams over multiuser OFDM networks. 


\section{A. Video Source Codec Subsystem}

The EWV encoder consists of four stages [16], namely, 3-D wavelet transform, quantization, bit plane arithmetic coding, and rate-distortion optimization. At the first stage, we collect a group of frames (GOF) as an encoding unit and apply 1-D dyadic temporal decomposition to obtain temporal subbands. The 2-D spatial dyadic decomposition is applied in each temporal subband to obtain wavelet spatiotemporal subbands (or "subbands" for short). At the second stage, a uniform quantizer is used for all wavelet coefficients in all subbands. At the third stage, fractional bit plane arithmetic coding is applied to each subband. Except that the most significant bit plane (MSB) has only one coding pass, every bit plane is encoded into three coding passes, namely, significance propagation pass, magnitude refinement pass, and normalization pass. Each coding pass can be treated as a candidate truncation point and the EWV decoder can decode the truncated bitstream containing an integer number of coding passes in each subband. The more consecutive coding passes of each subband a receiver receives, the higher decoded video quality we have. The coding passes among all subbands can be further grouped into several quality layers such that the received video quality can be refined progressively by receiving more layers. At the last stage, the encoder determines which coding passes are included in the output bit stream subject to quality or rate constraint.

To maintain the coding efficiency, the R-D curve in each subband should be convex [17]. Some coding passes in a subband cannot serve as feasible truncation points to maintain the convexity and they will be pruned from the truncation point list. To facilitate the discussion, we call all the coding passes between two truncation points as a coding pass cluster.

Consider now there are a total of $B$ subbands for the $k^{t h}$ user and the subband $b$ has $T_{k}^{b, \max }$ coding pass clusters. We can measure the rate and the corresponding decrease in normalized mean squared distortion of the $t^{t h}$ coding pass cluster in subband $b$ for the $k^{t h}$ user [17], and denote them as $\Delta r_{t, b, k}$ and $\Delta d_{t, b, k}$, respectively. We divide the whole duration for transmitting a total of $L$ quality layers into $L$ transmission intervals with equal length. The $l^{\text {th }}$ quality layer is transmitted at the $l^{\text {th }}$ transmission 


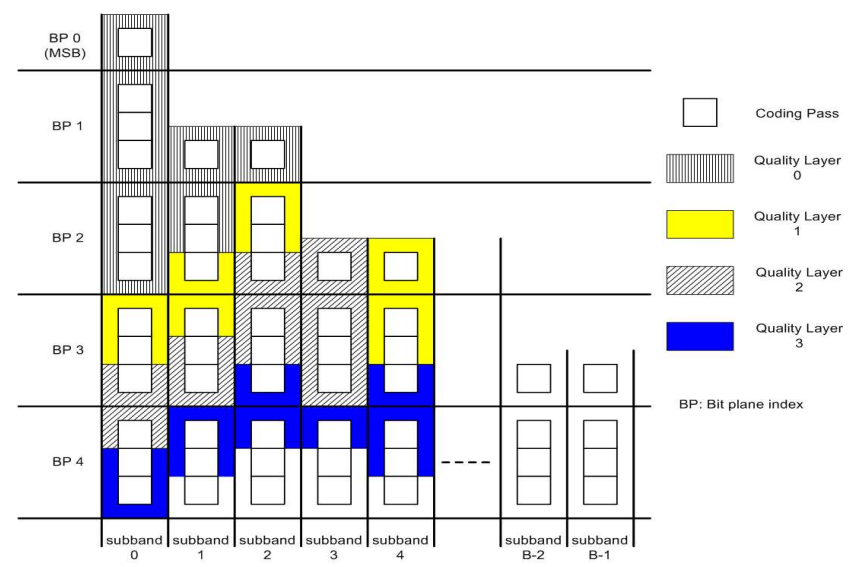

Fig. 1. Illustration of the relationship among coding pass, subband, and quality layer.

interval. The received distortion $D_{k}^{l}$ and rate $R_{k}^{l}$ for quality layers $O$ to $l$ can be expressed as:

$$
\begin{aligned}
& D_{k}^{l}=D_{k}^{\max }-\sum_{b=0}^{B-1} \sum_{t=0}^{T_{k}^{b, l}-1} \Delta d_{t, b, k}, \\
& R_{k}^{l}=\sum_{q=0}^{l} \Delta R_{k}^{q} .
\end{aligned}
$$

Here $D_{k}^{\max }$ is the distortion without decoding any coding pass cluster,

$$
\Delta R_{k}^{l}=\sum_{b=0}^{B-1} \sum_{t=T_{k}^{b, l-1}}^{T_{k}^{b, l}-1} \Delta r_{t, b, k},
$$

and $T_{k}^{b, l}$ is the total number of coding pass clusters of subband $b$ in the quality layers $O$ to $l$, which satisfies:

$$
0 \leq T_{k}^{b, l-1} \leq T_{k}^{b, l} \leq T_{k}^{b, \max }, \quad \forall b \text { and } 0<l<L .
$$

Define the number of coding pass clusters for subband $b$ in quality layer $l$ as $\Delta T_{k}^{b, l}=T_{k}^{b, l}-T_{k}^{b, l-1}$ and for all subbands

$$
\Delta \mathbf{T}_{k}^{l}=\left[\Delta T_{k}^{0, l}, \Delta T_{k}^{1, l}, \ldots, \Delta T_{k}^{B-1, l}\right]
$$

We also define a matrix $\Delta \mathbf{T}^{l}$ whose $k^{t h}$ row is $\Delta \mathbf{T}_{k}^{l}$. Thus, in each transmission interval $l$, the source coding part of our system determines the coding pass cluster assignment $\Delta \mathbf{T}_{k}^{l}$ and packetizes them as a quality layer for each user. We use Figure 1 to illustrate the relationship among coding pass, subband, and quality layer. Note that owing to different content complexities and motion activities shown in video sources, the R-D information should be evaluated for each GOF of each user to capture the characteristics of the corresponding bitstream. 


\section{B. Multiuser OFDM Subsystem}

We consider a downlink scenario of a single-cell multiuser OFDM system in which there are $K$ users randomly located. The system has $N$ subcarriers and each subcarrier has bandwidth of $W$. We use an indicator $a_{k n} \in\{0,1\}$ to represent whether the $n^{t h}$ subcarrier is assigned to user $k$. Note that in a singlecell OFDM system, each subcarrier can be assigned to at most one user, i.e., $\sum_{k=0}^{K-1} a_{k n} \in\{0,1\}, \forall n$. The overall subcarrier-to-user assignment can be represented as a matrix $\mathbf{A}$ with $[\mathbf{A}]_{k n}=a_{k n}$. Let $r_{k n}$ be the $k^{t h}$ user's transmission rate at the $n^{t h}$ subcarrier and the total rate for the $k^{t h}$ user can be expressed as $\sum_{n=0}^{N-1} a_{k n} r_{k n}$. The overall rate allocation can also be represented as a matrix $\mathbf{R}$ with $[\mathbf{R}]_{k n}=r_{k n}$.

In mobile wireless communication systems, signal transmission suffers from various impairments such as frequency-selective fading due to multipath delay [40]. The continuous complex baseband representation of user $k$ 's wireless channel impulse response is expressed as

$$
g_{k}(\mathrm{t}, \tau)=\sum_{i} v_{k, i}(\mathrm{t}) \delta\left(\tau-\tau_{k, i}\right),
$$

where $v_{k, i}(\mathrm{t})$ and $\tau_{k, i}$ are the gain and the delay of path $i$ for user $k$, respectively. In Rayleigh fading, the sequence $v_{k, i}(\mathrm{t})$ is modelled as a zero-mean circular symmetric complex Gaussian random variable with variance $\sigma_{v_{k, i}}^{2}$ proportional to $d^{-\alpha}$, where $d$ is the distance and $\alpha$ is the propagation loss factor. All $v_{k, i}(\mathrm{t})$ are assumed to be independent for different paths. The root-mean-square (RMS) delay spread is the square root of the second central moment of the power delay profile:

$$
\sigma_{k, \tau}=\sqrt{\overline{\tau_{k}^{2}}-\left(\overline{\tau_{k}}\right)^{2}}
$$

where $\overline{\tau_{k}^{2}}=\frac{\sum_{i} \sigma_{v_{k, i}}^{2} \tau_{k, i}^{2}}{\sum_{i} \sigma_{v_{k, i}}^{2}}$ and $\overline{\tau_{k}}=\frac{\sum_{i} \sigma_{v_{k, i}}^{2} \tau_{k, i}}{\sum_{i} \sigma_{v_{k, i}}^{2}}$.

After sampling at the receiver, the channel gain of OFDM subcarriers can be approximated by the discrete samples of the continuous channel frequency response as

$$
G_{k n}^{h}=\left.\int_{-\infty}^{\infty} g_{k}(\mathrm{t}, \tau) e^{-j 2 \pi f \tau} d \tau\right|_{f=n W, \mathrm{t}=h T_{f}},
$$

where $T_{f}$ is the duration of an OFDM symbol and $h$ is the sampling index. This approximation does not consider the effect of the smoothing filter at the transmitter and the front-end filter at the receiver. 
We assume a slow fading channel where the channel gain is stable within each transmission interval. ${ }^{1}$ The resource allocation procedure will be performed in each transmission interval. To facilitate the presentation, we omit $h$ in the channel gain notation. The channel parameters from different subcarrier of different users are assumed perfectly estimated, and the channel information is reliably fed back from mobiles users to the base station in time for use in the corresponding transmission interval. Denote $\Gamma_{k n}$ as the $k^{t h}$ user's signal to noise ratio (SNR) at the $n^{\text {th }}$ subcarrier as:

$$
\Gamma_{k n}=P_{k n} G_{k n} / \sigma^{2},
$$

where $P_{k n}$ is the transmission power for the $k^{t h}$ user at the $n^{t h}$ subcarrier and $\sigma^{2}$ is the thermal noise power that is assumed to be the same for each subcarrier of different users. Further, let $[\mathbf{G}]_{k n}=G_{k n}$ be the channel gain matrix and $[\mathbf{P}]_{k n}=P_{k n}$ the power allocation matrix. For downlink system, because of the practical constraints in implementation, such as the limitation of power amplifier and consideration of cochannel interferences to other cells, the overall power is bounded by $P_{m a x}$, i.e., $\sum_{k=0}^{K-1} \sum_{n=0}^{N-1} a_{k n} P_{k n} \leq$ $P_{\max }$.

The goal of the proposed framework is to provide good subjective video quality of the reconstructed video. Since the distortion introduced by channel error is typically more annoying than the distortion introduced by source lossy compression, the system should keep the channel-induced distortion at a negligible portion of the end-to-end distortion so that the video quality is controllable by the source coding subsystem. This can be achieved when we apply an appropriate amount of channel coding to keep the bit error rate (BER) after the channel coding below some targeted BER threshold [31], which is $10^{-6}$ in our system and achievable in most $3 \mathrm{G} / 4 \mathrm{G}$ systems. In addition, joint consideration of adaptive modulation, adaptive channel coding, and power control can provide each user with the ability to adjust each subcarrier's data transmission rate $r_{k n}$ to control video quality while meeting the required BER.

We focus our attention on MQAM modulation and convolutional codes with bit interleaved coded modulation (BICM) as they provide high spectrum efficiency and strong forward error protection, re-

\footnotetext{
${ }^{1}$ In practice, the duration of a transmission interval can be adjusted shorter enough so that the channel gain is stable within a transmission interval.
} 
TABLE I

REQUIRED SNR AND TRANSMISSION RATE USING ADAPTIVE MODULATION AND CONVOLUTIONAL CODING RATES [35]

\begin{tabular}{c|c|c|c|c|c}
\hline$k$ & Rate & Modulation & $\begin{array}{c}\text { Convolutional } \\
\text { Coding Rate }\end{array}$ & $\begin{array}{c}\text { SNR } \rho_{k}(\mathrm{~dB}) \text { for } \\
\text { BER } \leq 10^{-6}\end{array}$ & $\begin{array}{c}\text { SNR }(\mathrm{dB}) \text { for } \\
\text { BER } \leq 10^{-5}\end{array}$ \\
\hline 1 & $1 \mathrm{~W}$ & QPSK & $1 / 2$ & 4.65 & 4.09 \\
2 & $1.33 \mathrm{~W}$ & QPSK & $2 / 3$ & 6.49 & 5.86 \\
3 & $1.5 \mathrm{~W}$ & QPSK & $3 / 4$ & 7.45 & 6.84 \\
4 & $1.75 \mathrm{~W}$ & QPSK & $7 / 8$ & 9.05 & 8.44 \\
5 & $2 \mathrm{~W}$ & $16 \mathrm{QAM}$ & $1 / 2$ & 10.93 & 10.04 \\
6 & $2.66 \mathrm{~W}$ & 16QAM & $2 / 3$ & 12.71 & 12.13 \\
7 & $3 \mathrm{~W}$ & $16 \mathrm{QAM}$ & $3 / 4$ & 14.02 & 13.29 \\
8 & $3.5 \mathrm{~W}$ & 16QAM & $7 / 8$ & 15.74 & 15.01 \\
9 & $4 \mathrm{~W}$ & 64QAM & $2 / 3$ & 18.50 & 17.70 \\
10 & $4.5 \mathrm{~W}$ & 64QAM & $3 / 4$ & 19.88 & 18.99 \\
11 & $5.25 \mathrm{~W}$ & 64QAM & $7 / 8$ & 21.94 & 21.06 \\
\hline
\end{tabular}

spectively. We list the required SNRs' and the adopted modulation with convolutional coding rates to achieve different supported transmission rates under different BER requirement in Table I based on the results in [35]. Given a targeted BER, there is a one-to-one mapping between the selected transmission rate and the chosen modulation scheme with convolutional coding rate when the required SNR is satisfied. In this case, determining $r_{k n}$ is equal to determine the modulation and channel coding rate. For each rate allocation $[\mathbf{R}]_{k n}$, the corresponding power allocation $[\mathbf{P}]_{k n}$ should maintain the SNR in (9) larger than the corresponding value listed in Table I to achieve the BER requirement. To facilitate our discussion, we define the feasible set of the transmission rate in Table $\mathrm{I}$ as $\nu=\left\{\nu_{0}, \nu_{1}, \nu_{2}, \ldots, \nu_{Q}\right\}$ and the corresponding set of the required SNR for BER $\leq 10^{-6}$ as $\rho=\left\{\rho_{0}, \rho_{1}, \rho_{2}, \ldots, \rho_{Q}\right\}$. Here, $\nu_{0}=0$ and $\rho_{0}=0$, and $Q$ represents the number of combinations for different modulation with convolutional coding rates, which is 11 in our case. All transmission rate $r_{k n} \mathrm{~s}^{\prime}$ should be selected from the feasible rate set $\nu$.

\section{EWV Video over Multiuser OFDM}

The block diagram of the proposed wireless video system is shown in Figure 2. The upper and lower parts show the modules located in the server side and the mobile user side, respectively. For the server side, the server buffers each user's incoming video frames in the user's frame buffer. After collecting a GOF with $H$ frames for each user, the server moves those raw video frames to a wavelet video encoder for 


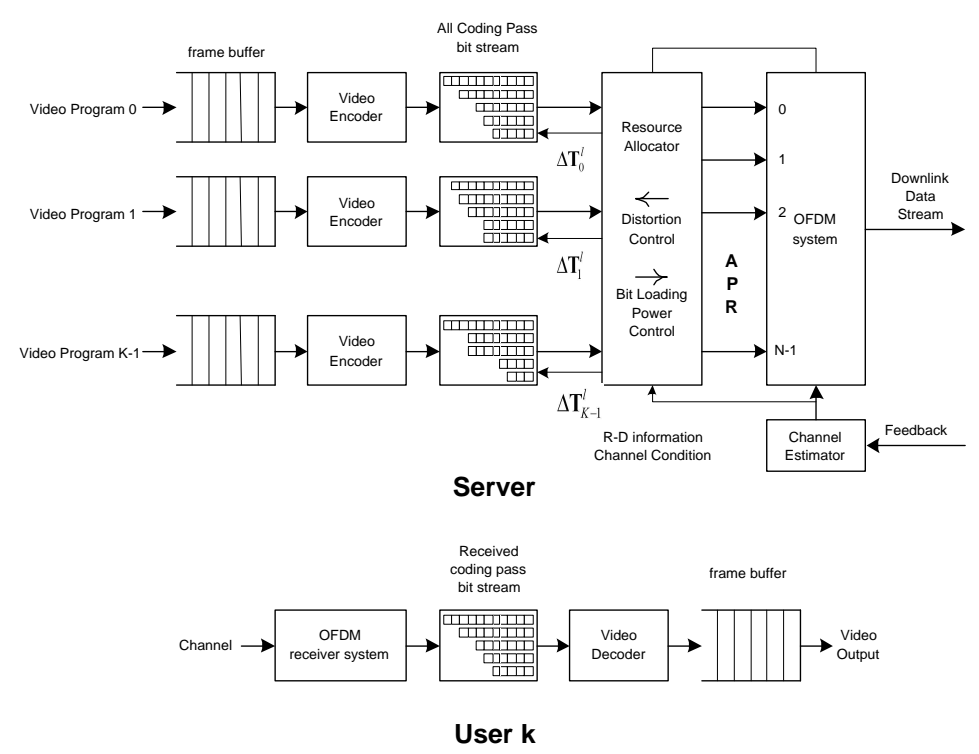

Fig. 2. System block diagram

compression as a coding pass bit stream. The selected coding pass clusters will be transmitted during the next GOF transmission time of $H / F$ second long, where $F$ is the video frame sampling rate. To capture the varying channel conditions and video content characteristics, the resource allocator should obtain the channel information for each transmission interval from the channel estimator and the R-D information of each GOF from the video coder. With the estimated channel conditions, the resource allocator can predict how many data rates with BER $\leq 10^{-6}$ the wireless networks can support in the next transmission interval. With the R-D information, the resource allocator can estimate the qualities of the reconstructed videos after decoding at each mobile terminal. By jointly considering the R-D information and the estimated channel conditions, the resource allocator performs resource optimization and distributes video and radio resources to each video stream in each transmission interval. According to the allocated resources, the source coding subsystem will group the selected coding pass clusters into a quality layer for each user and pass them to the transmission system; and the multiuser OFDM subsystem will load the video data to be transmitted to different subcarriers at a controlled amount of power. On the mobile user side, an OFDM receiver buffers the received data until the end of the current GOF transmission time. Then, those received data are moved to a wavelet video decoder for decoding and the decoded frames are sent for display during the next GOF transmission time. 
Since we only know the channel conditions provided by the channel estimator in the near future within the next transmission interval and the GOF bitstreams are transmitted across $L$ transmission intervals, it is necessary to break down the optimization problem into a sequential optimization problem and solve each problem in each transmission interval. There are two different objectives we want to achieve in each transmission interval: fairness and efficiency. To ensure the fairness among all users, we formulate the problem as a min-max optimization problem to minimize the maximal (weighted) end-toend distortion among all users. Maintaining short-term fairness in each transmission interval ensures the long-term fairness for GOFs [34]. To achieve high resource-allocation efficiency in terms of a high overall video quality, we formulate the problem as an optimization problem to minimize the overall end-to-end distortion among all users. We will discuss the fairness and efficiency problems in Section III and Section IV, respectively. The tradeoff between efficiency and fairness will be addressed in Section V.

\section{Optimization in Resource Allocator: Focusing on FAirness}

In this section, we consider how to achieve fair video quality among all users in a transmission interval and formulate this problem as a min-max problem. Given the integer programming nature of the problem, we propose a three-stage suboptimal algorithm to solve the optimization problem in real time.

\section{A. Formulation of the Fairness Problem}

At the beginning of the $l^{\text {th }}$ transmission interval, according to the channel information and subject to the transmission delay constraint as one transmission interval long, the resource allocator minimizes the maximal distortion received among all users as follows:

$$
\min _{\mathbf{A}, \mathbf{R}, \Delta \mathbf{T}^{l}} \max _{k} w_{k} \cdot f\left(D_{k}^{l}\right)
$$

$$
\text { subject to }\left\{\begin{array}{l}
\text { Subcarrier Assignment: } \sum_{k=0}^{K-1} a_{k n} \leq 1, a_{k n} \in\{0,1\}, \forall n ; \\
\text { Subcarrier Rate: } r_{k n} \in \nu, \quad \forall k, n ; \\
\text { User Rate: } 0 \leq \Delta R_{k}^{l} \leq \sum_{n=0}^{N-1} a_{k n} r_{k n}, \forall k \\
\text { Power: } \sum_{k=0}^{K-1} \sum_{n=0}^{N-1} a_{k n} P_{k n} \leq P_{\max }
\end{array}\right.
$$

where $w_{k}$ is the quality weighting factor and $f(\cdot)$ the perceptual distortion function. We solve this optimization problem by selecting the values of subcarrier assignment matrix $\mathbf{A}$, rate assignment matrix 


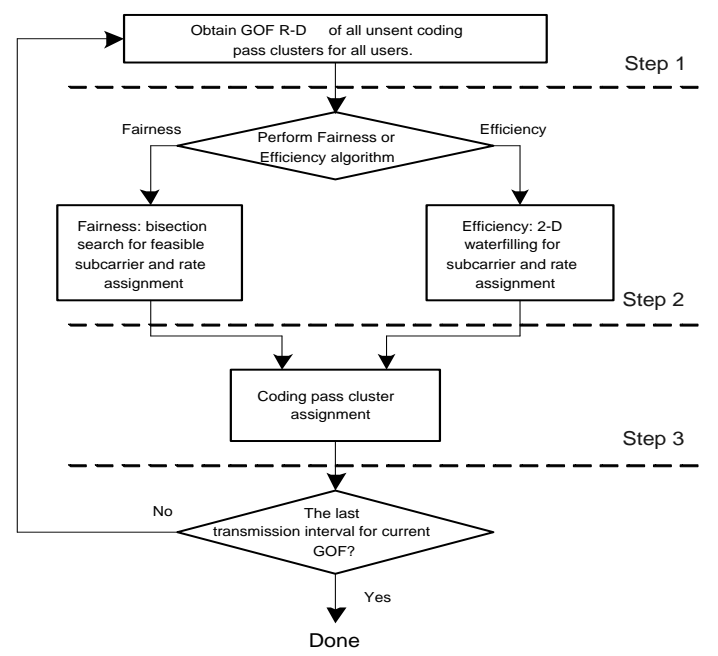

Fig. 3. Flowchart of the proposed algorithm

$\mathbf{R}$, and coding pass cluster assignment $\Delta \mathbf{T}^{l}$ subject to four constraints: the first constraint is on subcarrier assignment that a subcarrier can be assigned to at most one user; the second one restricts the subcarrier rate to be selected only from the feasible rate set $\nu$; the third one is that the user's overall assigned rate in (3) should be no larger than the overall assigned subcarrier rate; and the fourth one is on the maximal power available for transmission. Note that the system can provide differentiated service by setting $\left\{w_{k}\right\}$ to different values according to the quality levels requested by each user. As a proof of concept, we consider the case of $w_{k}=1, \forall k$ and $f\left(D_{k}^{l}\right)=D_{k}^{l}$ for providing uniform quality among all users here. The proposed solution can be easily extended to other quality weighting factors and quality functions, and we will demonstrate the ability for providing differentiated service in Section VI. The problem in (10) is a multi-dimension generalized assignment problem, which is an $N P$ hard problem [36]. In a real-time system, a fast approximation algorithm with good performance is needed and will be designed next.

\section{B. Proposed Algorithm for Fairness}

We propose a three-stage fast algorithm to solve the optimization problem (10). As illustrated by the flowchart in Figure 3, at the first stage, we obtain continuous GOF R-D functions that provide a distortionto-rate mapping to facilitate the resource allocation. At the second stage, we determine the subcarrier assignment matrix $\mathbf{A}$ and rate assignment matrix $\mathbf{R}$ to find the largest distortion reduction that the OFDM system can support. This goal can be achieved through a bisection search on the R-D functions obtained 
TABLE II

GOF R-D USED IN EACH TRANSMISSION INTERVAL

\begin{tabular}{|l|}
\hline a) Sort all $\left\{\lambda_{t, b, k}\right\}$ for all $t \geq T_{k}^{b, l-1}$ in a decreasing order for user $k$ \\
\hline b) For each $(t, b)$ for user $k$, we have indices \\
$I_{k}(t, b) \in\left\{1,2, \ldots, M_{k}^{l}\right\}$ and $I_{k}^{-1}(m) \in\{(t, b)\}$ \\
s.t. $\lambda_{i, b, k}>\lambda_{j, c, k}$ for $I_{k}(i, b)<I_{k}(j, c)$ \\
\hline c) Set $D_{k}^{l}[0]=D_{k}^{l-1}$ and $R_{k}^{l}[0]=0$ \\
For $m=1, \ldots, M_{k}^{l}$ \\
$\quad D_{k}^{l}[m]=D_{k}^{l}[m-1]-\left|\Delta d_{I_{k}^{-1}(m), k}\right|$ \\
$\quad R_{k}^{l}[m]=R_{k}^{l}[m-1]+\Delta r_{I_{k}^{-1}(m), k}$ \\
d) Construct the continuous GOF R-D function \\
$D_{k}^{l}\left(\gamma_{k}^{l}\right)=\frac{D_{k}^{l}[m+1]-D_{k}^{l}[m]}{R_{k}^{l}[m+1]-R_{k}^{l}[m]}\left(\gamma_{k}^{l}-R_{k}^{l}[m]\right)+D_{k}^{l}[m]$, \\
for $R_{k}^{l}[m] \leq \gamma_{k}^{l} \leq R_{k}^{l}[m+1]$ and $m=0, \ldots, M_{k}^{l}-1$. \\
\hline
\end{tabular}

at Stage 1. At the third stage, the coding pass cluster assignment $\Delta \mathbf{T}^{l}$ is decided subject to the allocated subcarrier and rate assignment at Stage 2. We explain the details of each stage below:

1) Stage 1: At this stage, a continuous GOF R-D function of the unsent coding pass clusters for each user is obtained. The goal for determining the GOF R-D function is to provide a one-to-one mapping between rate and distortion such that we can know the amount of rate increment necessary for a given amount of reduction in distortion.

Suppose there are $M_{k}^{l}$ unsent coding pass clusters for user $k$ at the beginning of the transmission interval $l$. Define the distortion-to-length slope for a coding pass cluster with the rate increment $\Delta r_{t, b, k}$ and the distortion reduction $\Delta d_{t, b, k}$ as

$$
\lambda_{t, b, k} \triangleq\left|\Delta d_{t, b, k}\right| / \Delta r_{t, b, k}
$$

The distortion-to-length slope represents how much distortion a coding pass cluster can reduce by given one unit of rate. We can sort all distortion-to-length slopes of all unsent coding pass clusters $\left(\lambda_{t, b, k}\right.$, where $T_{k}^{b, l-1} \leq t$ ) in a decreasing order and obtain the corresponding mapping indices $I_{k}(t, b)$ and inverse indices $I_{k}^{-1}(m) \in\{(t, b)\}$. For example, if the sorting result is $\lambda_{t_{1}, b_{1}, k}>\lambda_{t_{2}, b_{2}, k}>\cdots$, we assign $I_{k}\left(t_{1}, b_{1}\right)=1, I_{k}\left(t_{2}, b_{2}\right)=2$, and $I_{k}^{-1}(1)=\left(t_{1}, b_{1}\right), I_{k}^{-1}(2)=\left(t_{2}, b_{2}\right)$. Then, a decreasing discrete R-D function $\left(R_{k}^{l}[m], D_{k}^{l}[m]\right)$ for quality layer $l$ can be obtained according to this sorting result, as shown in Table II (c). To facilitate the distortion-to-rate searching, we relax the constraints on integer value of rate and integer number of coding pass clusters to allow them to be real numbers; and construct 
a continuous R-D function through linear interpolation of the discrete R-D function as follows:

$$
\begin{aligned}
& D_{k}^{l}\left(\gamma_{k}^{l}\right)=\frac{D_{k}^{l}[m+1]-D_{k}^{l}[m]}{R_{k}^{l}[m+1]-R_{k}^{l}[m]}\left(\gamma_{k}^{l}-R_{k}^{l}[m]\right)+D_{k}^{l}[m], \\
& \text { for } R_{k}^{l}[m] \leq \gamma_{k}^{l} \leq R_{k}^{l}[m+1] \text { and } m=0, \ldots, M_{k}^{l}-1,
\end{aligned}
$$

where $\gamma_{k}^{l}$ is the required bit rate. We can calculate the least required rate, $\gamma_{k}^{l}$, to achieve the targeted distortion, $D$, by finding the inverse function of $D_{k}^{l}(\cdot)$. We summarize the algorithm used in this stage in Table II. The complexity of this stage for each user is $O\left(M_{k}^{l} \log \left(M_{k}^{l}\right)\right)$ due to sorting.

2) Stage 2: At this stage, the goal is to minimize the maximal distortion supported by the OFDM subsystem through a bisection search procedure. By checking the continuous GOF R-D functions obtained in (12), the resource allocator can calculate the minimum transmission rates $\left\{\gamma_{k}^{l}\right\}$ necessary to achieve the same targeted distortion among all users. Then the resource allocator checks whether these requested rates can be supported by the OFDM subsystem under current channel conditions. If the requested rates are feasible, the resource allocator tries to further decrease the targeted distortion by increasing the requested rates. Otherwise, the resource allocator increases the targeted distortion to reduce the requested rates and checks the feasibility again. A bisection search algorithm is deployed to find the minimal distortion $D$ that the OFDM subsystem can support.

The feasibility of the requested rates depends on two factors. First, the OFDM subsystem should be able to transmit the requested rates $\left\{\gamma_{k}^{l}\right\}$ for all users. Second, the overall transmission power, $P_{\text {sum }}$, cannot exceed $P_{\max }$. We develop a fast suboptimal algorithm shown in Table III to allocate the bits and power to satisfy the rate constraint first and then the power constraint. There are three steps in the proposed algorithm for feasibility checking: initialization, minimal rate assignment, and power reduction. First, the subcarrier assignment matrix $\mathbf{A}$, the rate assignment matrix $\mathbf{R}$, and the power assignment matrix $\mathbf{P}$ are initialized to zeros. Next, the system tries to satisfy the requested rates. In each round, we allocate an unassigned subcarrier to a user. If $G_{\hat{k} \hat{n}}$ has the maximal value in current $\mathbf{G}$, subcarrier $\hat{n}$ is assigned to user $\hat{k}$ and we update $G_{k \hat{n}}=0, \forall k$ to prevent this subcarrier from being assigned again. We then determine the modulation schemes and the coding rates for all subcarriers currently allocated to user $\hat{k}$ such that the requested data rate can be accommodated and the required transmission power is minimized 
TABLE III

\section{Resource Allocation AND FEASibiLity CHECK In OFDM SUbSystem}

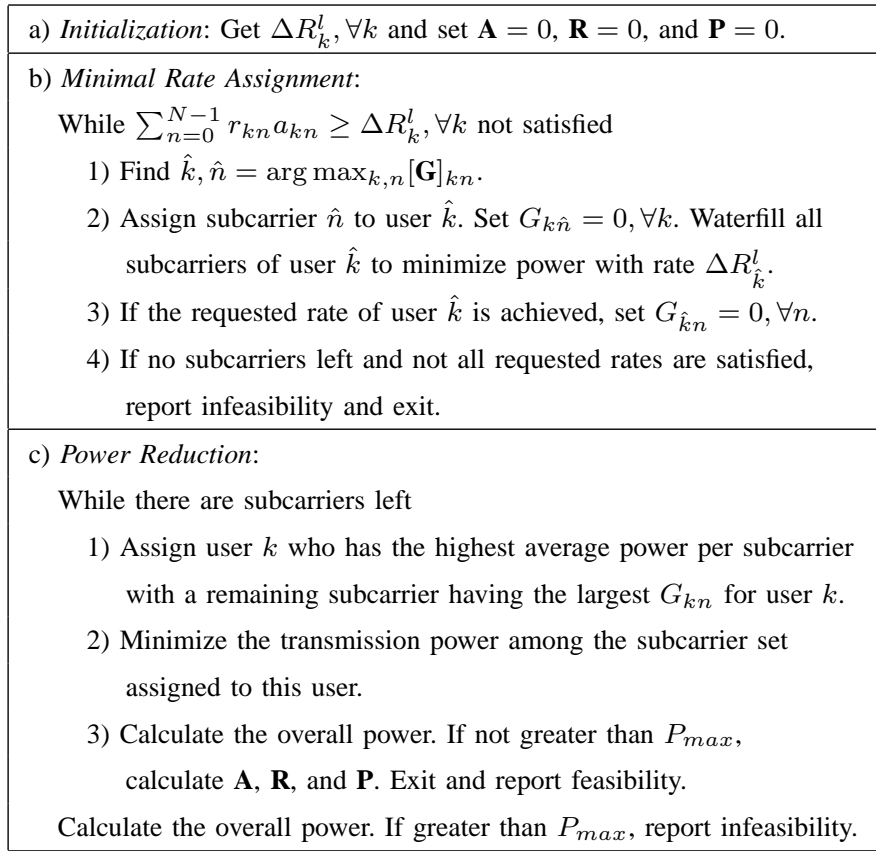

in the meantime. This can be implemented by the well-known waterfilling algorithm with Table I and (9). If the requested rate of user $\hat{k}$ can be allocated, user $\hat{k}$ is removed from future assignment list in this step by assigning $G_{\hat{k} n}=0, \forall n$. This step is repeated until all users' requested rates are satisfied. If all subcarriers are already assigned and not all requested rates can be allowed, infeasibility is reported and the resource allocator has to reduce the requested rates. In the third step, we try to reduce the overall transmission power $P_{\text {sum }}$ to be below $P_{\max }$ by assigning the remaining subcarriers. In each round, we select a user who has the highest average power per subcarrier and assign him/her with one of the remaining subcarriers in which this user has the largest channel gain. Then we minimize the transmission power among the subcarrier set assigned to this user. The overall transmission power is calculated and if it is greater than $P_{\max }$, the power reduction procedure is repeated again. Otherwise, we calculate $\mathbf{A}$, $\mathbf{R}$, and $\mathbf{P}$ for OFDM subsystem, report feasibility and exit. An infeasibility is reported if there is no subcarrier left and $P_{\text {sum }}>P_{\max }$. Since the required power for each user in each subcarrier can be pre-calculated, the complexity of checking feasibility in each iteration is $O(N)$. The overall number of iterations, which is typically fewer than 20 in our experiment, is bounded by the bisection search. 
TABLE IV

CODING PASS CLUSTER ASSIGNMENT

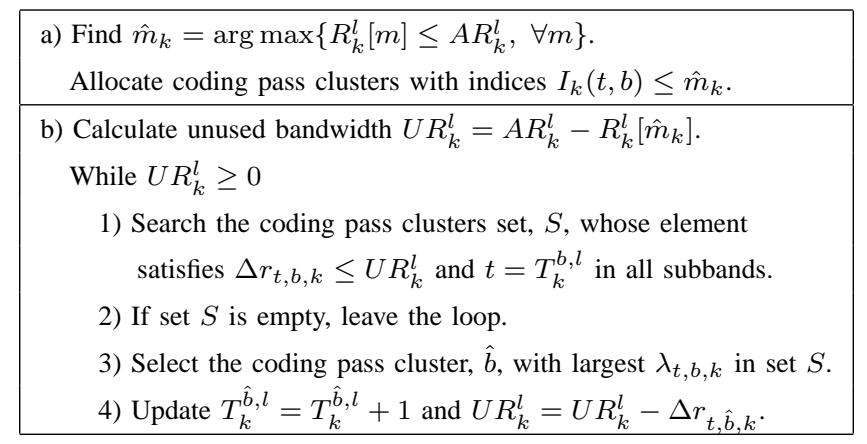

3) Stage 3: At this stage, we perform the coding pass cluster assignment for each user individually. Denote the assigned rate from Stage 2 for the $k^{t h}$ user as $A R_{k}^{l}=\sum_{n=0}^{N-1} a_{k n} r_{k n}$. Due to the discrete rate provided by the OFDM subsystem $\left(r_{k n} \in \nu, \forall k, n\right)$, the assigned transmission rate $A R_{k}^{l}$ is generally larger than the requested rate, i.e., $A R_{k}^{l} \geq \gamma_{k}^{l}$. Therefore, we have rate budget $A R_{k}^{l}$ to allocate the coding pass clusters. We formulate the problem as minimizing the distortion subject to the rate constraint by allocating the coding pass cluster:

$$
\min _{\Delta \mathbf{T}_{k}^{l}} D_{k}^{l} \text { subject to } \Delta R_{k}^{l} \leq A R_{k}^{l}
$$

Since for each unsent coding pass cluster we need to decide whether we select it or not, the problem (13) is a binary knapsack problem [36], which is $N P$ hard. To ensure the real-time performance, we apply a two-step greedy algorithm here. First, among all values of $R_{k}^{l}[m]$ that are not larger than $A R_{k}^{l}$, we find the largest one, $R_{k}^{l}\left[\hat{m}_{k}\right]$. We will include all coding pass clusters whose indices $I_{k}(t, b)$ are not larger than $\hat{m}_{k}$ in the current quality layer. Notice that the sorting order $\left\{I_{k}(t, b)\right\}$ has ensured the decoding dependency of coding pass clusters in each subband. This is because if user $k$ receives the $t^{\text {th }}$ coding pass cluster in subband $b$, user $k$ must receive coding pass cluster 0 to $t-1$ since $\lambda_{t^{\prime}, b, k}>\lambda_{t, b, k}, \forall t^{\prime}<t$ or $I_{k}\left(t^{\prime}, b\right)<I_{k}(t, b), \forall t^{\prime}<t$ due to the convexity of R-D in subband $b$. Second, a round of refinement is performed to utilize the unused bandwidth, $U R_{k}^{l}=A R_{k}^{l}-R_{k}^{l}\left[\hat{m}_{k}\right]$. We search all unsent coding pass clusters that follow the currently selected truncation points and pick those with rates not larger than the unused bandwidth. The coding pass cluster with the largest distortion-to-length slope is selected for transmission during current transmission interval. The system updates the unused bandwidth and unsent 
coding pass clusters; and then repeats the above search procedure until there is no coding pass cluster with size not larger than the unused bandwidth. Since the first step directly uses the result in (12), the refinement step consumes most computation power in the whole coding pass cluster assignment and the complexity to search a feasible coding pass cluster is $O(B)$. We recap the algorithm used in this stage in Table IV.

\section{Optimization in Resource Allocator: Focusing on EfFiciency}

In this section, we study the efficiency problem in which the overall distortion of all users is minimized in a transmission interval. We first formulate the efficiency problem as an optimization problem. Then, similar to the fairness case, we also propose a three-stage algorithm to determine the subcarrier assignment, rate assignment, and coding pass cluster assignment to achieve the optimization goal.

\section{A. Formulation of the Efficiency Problem}

We formulate the efficiency problem as to minimize the overall (weighted) end-to-end distortion among all users subject to constraints on subcarrier assignment, subcarrier rate, user rate, and power:

$$
\min _{\mathbf{A}, \mathbf{R}, \Delta \mathbf{T}^{l}} \sum_{k=0}^{K-1} w_{k} \cdot f\left(D_{k}^{l}\right)
$$

$$
\text { subject to }\left\{\begin{array}{l}
\text { Subcarrier Assignment: } \sum_{k=0}^{K-1} a_{k n} \leq 1, a_{k n} \in\{0,1\}, \forall n ; \\
\text { Subcarrier Rate: } r_{k n} \in \nu, \forall k, n ; \\
\text { User Rate: } 0 \leq \Delta R_{k}^{l} \leq \sum_{n=0}^{N-1} a_{k n} r_{k n}, \forall k ; \\
\text { Power: } \sum_{k=0}^{K-1} \sum_{n=0}^{N-1} a_{k n} P_{k n} \leq P_{\text {max }} .
\end{array}\right.
$$

The constraints are similar to the fairness case presented in Section III-A. Similar to the fairness case, the delay constraint is implicitly imposed in the problem (14) so as the transmission delay is restricted within a transmission interval.

\section{B. Proposed Algorithm for Efficiency}

To solve this minimization problem, we propose a three-stage algorithm shown in Figure 3 . The first stage is to obtain the continuous R-D functions of all unsent coding pass clusters for the current GOF. The second stage is to perform subcarrier assignment and rate assignment through a 2-D waterfilling 
procedure; and the third stage is the coding pass cluster assignment. The first and third stage are the same as what have been discussed in Section III-B. Here, we focus on the second stage and consider the case of $w_{k}=1, \forall k$ and $f\left(D_{k}^{l}\right)=D_{k}^{l}$.

Having the continuous R-D functions, the problem (14) can be simplified as follows:

$$
\min _{\mathbf{A}, \mathbf{R}} \sum_{k=0}^{K-1} D_{k}^{l}\left(\gamma_{k}^{l}\right)
$$

subject to $\left\{\begin{array}{l}\text { Subcarrier Assignment: } \sum_{k=0}^{K-1} a_{k n} \leq 1, a_{k n} \in\{0,1\}, \forall n ; \\ \text { Subcarrier Rate: } r_{k n} \in \nu, \forall k, n ; \\ \text { Power: } \sum_{k=0}^{K-1} \sum_{n=0}^{N-1} a_{k n} P_{k n} \leq P_{\text {max }} ;\end{array}\right.$

where $\gamma_{k}^{l}=\sum_{n=0}^{N-1} a_{k n} r_{k n}$. To solve this problem, a two-step suboptimal algorithm is proposed by first determining the subcarrier assignment matrix $\mathbf{A}$ and then deciding the rate assignment matrix $\mathbf{R}$.

1) Subcarrier Assignment: In this step, we relax the power constraint by assuming the maximal transmission power is unlimited and thus each subcarrier can be loaded with maximum rate $\nu_{Q}$ to fully utilize the available bandwidth. Then, the problem (15) has only the subcarrier assignment constraint and the goal is to find the subcarrier-to-user assignment that can reduce most distortion by using the least amount of power. This problem can be solved by an iterative greedy algorithm. In each iteration, we evaluate which user can achieve the most distortion reduction by using the least power if we assign an unused subcarrier to him/her. There are two factors affecting this evaluation, as reflected by $\psi_{k n}$ and defined below:

$$
\psi_{k n} \triangleq\left(\frac{D_{k}^{l}\left(\gamma_{k}^{l}\right)-D_{k}^{l}\left(\gamma_{k}^{l}+\nu_{Q}\right)}{\nu_{Q}}\right)\left(\frac{\nu_{Q}}{\frac{\rho_{Q} \sigma^{2}}{G_{k n}}}\right) .
$$

$\gamma_{k}^{l}$ is the accumulative allocated rate for user $k$ in the current iteration. The first term of (16) evaluates the gradient of reduced video distortion with respect to the allocated rate, i.e., how much distortion we can reduce by assigning a unit of rate for user $k$. The second term of (16) evaluates the gradient of the allocated rate to the required transmission power (calculated using (9)), i.e., how many bits this system can transmit at $\mathrm{BER} \leq 10^{-6}$ per unit of power. If both factors of user $k$ at subcarrier $n$ are large, it implies that assigning subcarrier $n$ to user $k$ can use the same amount of power to reduce more distortion. Since the second term is only a function of channel gain, we can further simplify (16) and have a matrix $\Psi$ 
as $[\boldsymbol{\Psi}]_{k n}=\psi_{k n}$ :

$$
\psi_{k n}=\left(D_{k}^{l}\left(\gamma_{k}^{l}\right)-D_{k}^{l}\left(\gamma_{k}^{l}+\nu_{Q}\right)\right) G_{k n}
$$

Once we obtain $\Psi$, we can find its entry $(\hat{k}, \hat{n})$ with maximal value and assign subcarrier $\hat{n}$ to user $\hat{k}$. To prevent this subcarrier from being assigned again, we set $G_{k \hat{n}}=0, \forall k$. Then, we update the current allocated rate of user $\hat{k}$ by setting $\gamma_{\hat{k}}^{l}=\gamma_{\hat{k}}^{l}+\nu_{Q}$ and subcarrier assignment matrix by setting $a_{\hat{k} \hat{n}}=1$ and $a_{k^{\prime} \hat{n}}=0$ for $k^{\prime} \neq \hat{k}$. This procedure is repeated until all subcarriers are assigned. The complexity of this step is $O(N)$.

2) Rate Assignment: Based on the subcarrier assignment in the previous step, we determine how much rate should be assigned to each subcarrier. To facilitate our discussion, let $\theta_{k n} \in\{0,1, \ldots Q\}$ be the subcarrier usage index, which indicates the selected row in Table I for user $k$ at subcarrier $n$. For example, $\theta_{k n}=q$ represents that user $k$ has loaded $r_{k n}=\nu_{q}$ bits in subcarrier $n$ and the required SNR to achieve BER $\leq 10^{-6}$ is $\rho_{q}$. Further, we define a set of incremental rate $\Delta \nu=\left\{\Delta \nu_{1}, \Delta \nu_{2}, \ldots, \Delta \nu_{Q}\right\}$ and a set of incremental power $\Delta \rho=\left\{\Delta \rho_{1}, \Delta \rho_{2}, \ldots, \Delta \rho_{Q}\right\}$, where $\Delta \nu_{q}=\nu_{q}-\nu_{q-1}$ and $\Delta \rho_{q}=\rho_{q}-\rho_{q-1}$ for $q=1, \ldots, Q$, respectively. We solve this rate assignment problem using a 2-D discrete waterfilling algorithm. At the beginning, we set all required rate $\left\{\gamma_{k}^{l}\right\}$ and all subcarrier usage indices $\left\{\theta_{k n}\right\}$ to zeros. In each iteration, similar to Step 1, we select the subcarrier setting that can achieve the most distortion reduction by using the least power when we evaluate the results of filling each subcarrier an incremental rate $\Delta \nu_{\theta_{k n}+1}$. This procedure is repeated until all subcarriers are fully loaded or the overall required power reaches the maximal available amount. The evaluation of distortion-to-power ratio for all subcarriers and users can be quantified as a matrix $\boldsymbol{\Phi}$ with $[\boldsymbol{\Phi}]_{k n}=\phi_{k n}$ :

$$
\phi_{k n} \triangleq\left(\frac{D_{k}^{l}\left(\gamma_{k}^{l}\right)-D_{k}^{l}\left(\gamma_{k}^{l}+\Delta \nu_{\theta_{k n}+1}\right)}{\Delta \nu_{\theta_{k n}+1}}\right)\left(\frac{\Delta \nu_{\theta_{k n}+1}}{\Delta \rho_{\theta_{k n}+1} \sigma^{2} / G_{k n}}\right) .
$$

The first term of (18) represents how much distortion user $k$ can reduce with an extra unit of rate and the second term of (18) represents how many bits to transmit for user $k$ at subcarrier $n$ with a unit of power. The overall $\phi_{k n}$ represents how much distortion user $k$ will reduce at subcarrier $n$ with one extra unit of power.

After obtaining $\boldsymbol{\Phi}$, we select the entry $(\hat{k}, \hat{n})$ with largest value. If subcarrier $\hat{n}$ does not belong to 
TABLE V

PROPOSED ALGORITHM TO MINIMIZE OVERALL DISTORTION

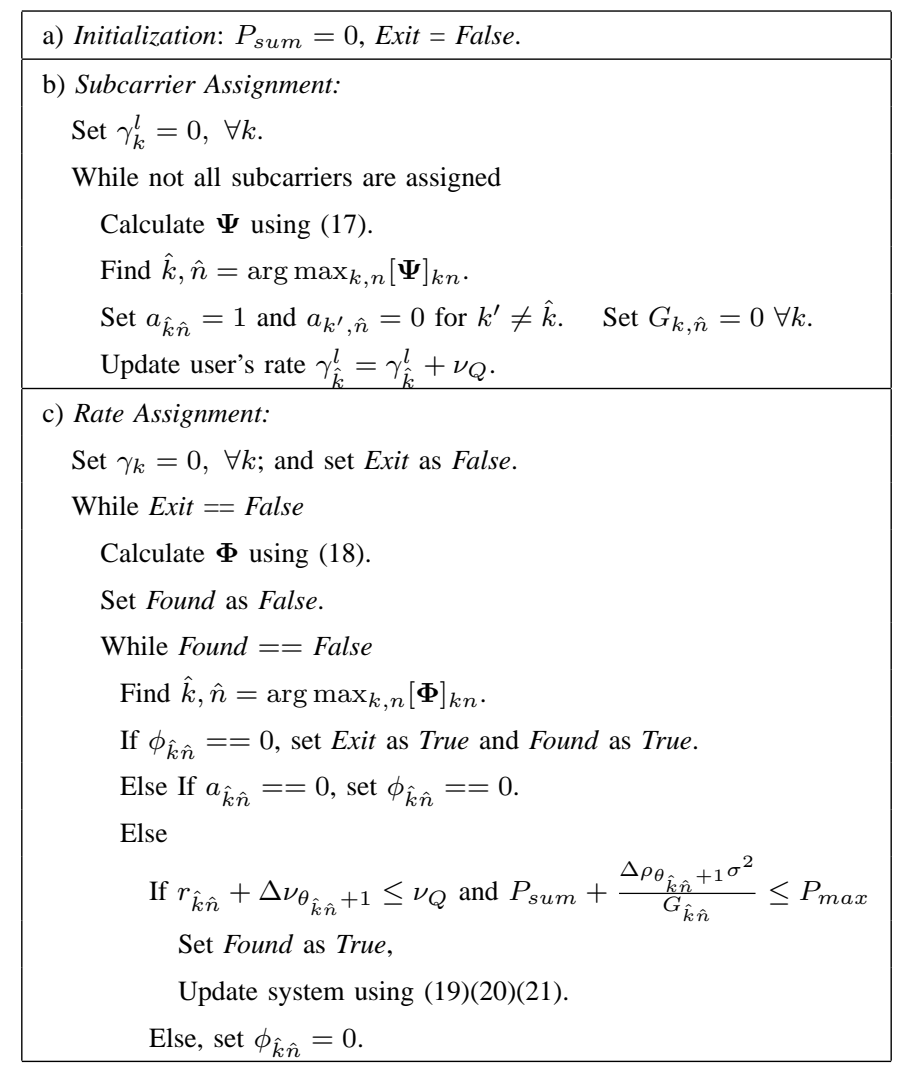

user $\hat{k}$, i.e., $a_{\hat{k} \hat{n}}=0$, we set $\phi_{\hat{k} \hat{n}}=0$ and search the highest value in $\Phi$ again. If so, we update user $\hat{k}$ 's subcarrier usage index

$$
\theta_{\hat{k} \hat{n}}=\theta_{\hat{k} \hat{n}}+1,
$$

the overall transmission rate of user $\hat{k}$

$$
\gamma_{\hat{k}}^{l}=\gamma_{\hat{k}}^{l}+\Delta \nu_{\theta_{\hat{k} \hat{n}}}
$$

and the overall transmission power

$$
P_{\text {sum }}=P_{\text {sum }}+\frac{\Delta \rho_{\theta_{\hat{k} \hat{n}}} \sigma^{2}}{G_{\hat{k} \hat{n}}} .
$$

If subcarrier $\hat{n}$ is overloaded, (i.e., $r_{\hat{k} \hat{n}}>\nu_{Q}$ ), or the overall required power exceeds the $P_{\max }$, we need to pick other entry by setting $\phi_{\hat{k} \hat{n}}=0$ and search the highest value in $\boldsymbol{\Phi}$ until a valid one is found. The search algorithm terminates if no more valid assignment is found. The whole algorithm is presented in Table V. Since the accumulative rate $\gamma_{\hat{k}}^{l}$ is updated for the selected user $\hat{k}$ only, the complexity of updating $\boldsymbol{\Phi}$ in each iteration is $O\left(n_{\hat{k}}\right)$, where $n_{\hat{k}}$ is the number of subcarriers assigned to user $\hat{k}$. The 
maximal number of iteration in the rate assignment is $N Q$; and the actual number of iteration depends on the transmission power level and channel condition.

\section{Extended Functionalities in the Proposed Framework}

In this section, we discuss the extended functionalities based on the proposed algorithms in Section III and IV. We first address how to achieve a desired tradeoff between system fairness and efficiency. Then we investigate how to incorporate unequal error protection in the proposed framework to increase system's efficiency.

\section{A. Tradeoff between Fairness and Efficiency}

We have proposed two solutions to ensure fairness and improve efficiency in each transmission interval, respectively. If we apply fairness algorithm in all transmission intervals ( $L$ intervals), the received video qualities for all users will be similar to each other. However, the users whose video programs require more rates to achieve the same video quality or who are in bad channel conditions will become a bottleneck in the whole system and degrade the overall video qualities. If we apply efficiency algorithm in all transmission intervals, the system will achieve the highest overall video qualities. Nevertheless, the users in good channel conditions with low video content complexity will be assigned more system resources. Consequently, some users will have unnecessarily good video qualities while others will have very bad qualities. In other words, a system achieving more efficiency will suffer from more unfairness. We are interested in how to design a system with partial fairness and partial efficiency. To achieve this tradeoff, for each GOF, we propose to apply fairness algorithm in the first several transmission intervals ( $x$ intervals) to ensure the baseline fairness, and then apply the efficiency algorithm in the rest transmission intervals ( $y=L-x$ intervals) to improve the overall video qualities. We denote this strategy as $\mathrm{F}_{x} \mathrm{E}_{y}$ algorithm. Note that $\mathrm{F}_{L} \mathrm{E}_{0}$ algorithm is the pure fairness algorithm and $\mathrm{F}_{0} \mathrm{E}_{L}$ algorithm is the pure efficiency algorithm. 


\section{B. Unequal Error Protection}

It has been shown that the unequal error protection (UEP) can improve the expected video qualities [37]-[39]. Relaxing the requirement from lower targeted BER to higher targeted BER but sending the same bit rate, the required power can be reduced. In other words, if the overall transmission power is fixed, the overall bit rate using higher targeted BER can be higher than the one with lower targeted BER. It is potential to improve the overall expected video qualities. The UEP takes the advantage of different priorities within a video bit stream by using different targeted BER. For the video bit stream with higher priority, the UEP adopts stronger error protection (lower targeted BER) to increase the probability of successful transmission. For the video bit stream with lower priority, the UEP applies weaker error protection (higher targeted BER) to utilize a larger effective bandwidth for statistical performance gain.

Because the EWV bit stream exhibits a strong decoding dependency, all received coding pass clusters in a subband should be adjacent to each other and also a truncated version of the original bit stream starting from MSB. Assuming all bits in the quality layer $0 \sim l-1$ are received correctly, given a targeted $\mathrm{BER}_{i}$, the expected distortion of the quality layer $l$ can be represented as:

$$
E_{i}\left[D_{k}^{l}\right]=D_{k}^{l-1}-\sum_{b=0}^{B-1} \sum_{t=T_{k}^{b, l-1}}^{T_{k}^{b, l}-1} p_{t, b, k}^{i} \Delta d_{t, b, k} .
$$

Here $p_{t, b, k}^{i}$ is the probability that the receiver can correctly receive all coding pass clusters from $T_{k}^{b, l-1}$ to $t$ in subband $b$ and can be expressed as follows:

$$
p_{t, b, k}^{i}=\left(1-\mathrm{BER}_{i}\right)^{\Delta R_{t, b, k}} .
$$

Here $\Delta R_{t, b, k}$ is the cumulative number of bits from coding pass cluster $T_{k}^{b, l-1}$ to $t$ in subband $b$ and can be expressed as

$$
\Delta R_{t, b, k}=\sum_{t^{\prime}=T_{k}^{b, l-1}}^{t} \Delta r_{t^{\prime}, b, k} .
$$

Quality layer $l$ has higher priority than quality layer $k$ if $l<k$ since both layers may have coding passes in the same subband so that coding passes in quality layer $k$ have decoding dependency on the ones in quality layer $l$ due to (4). We incorporate the unequal error protection strategy in the proposed framework by considering the priorities of quality layers in different transmission intervals. In the first 
$L-1$ transmission intervals, we solve the original problem (15) using the proposed algorithm shown in Table $\mathrm{V}$ with the strongest error protection. At the last transmission interval, we solve the problem (15) but replacing $D_{k}^{l}$ with $E_{i}\left[D_{k}^{l}\right]$ using several different $\mathrm{BER}_{i}$ as shown in Table I $\left(\mathrm{BER}_{0}=10^{-6}\right.$ and $\mathrm{BER}_{1}=10^{-5}$ in our case). We pick the BER setting that achieves the lowest overall expected distortion.

\section{Simulation Results}

The simulations are set up as follows. The OFDM system has 32 subcarriers over a total $1.6 \mathrm{MHz}$ bandwidth. The delay spread in root mean square is $3 \times 10^{-7} \mathrm{~s}$. An additional $5 \mu$ s guard interval is used to avoid inter-symbol interference due to channel delay spread. This results in a total block length as $25 \mu s$ and a block rate as $40 \mathrm{~K}$ per second. The Doppler frequency is $10 \mathrm{~Hz}$ and the transmission interval is $33.33 \mathrm{~ms}$. The mobile is uniformly distributed within the cell with radius of $50 \mathrm{~m}$ and the minimal distance from mobile to the base station is $10 \mathrm{~m}$. The noise power is $5 \times 10^{-9}$ Watts, and the maximal transmission power is 0.1 Watts. The propagation loss factor is 3 [40]. The video sampling rate is 30 frames per second with CIF resolution (352x288). The GOF size is 16 frames and each GOF is encoded by the codec [16] using Daubechies 9/7 bi-orthogonal filter with 4-level temporal decomposition and 3/2/1 spatial decomposition in low/mid/high temporal subbands, respectively.

\section{A. Performance Evaluation of the Fairness Algorithm}

We first demonstrate how the proposed algorithm $\mathrm{F}_{16} \mathrm{E}_{0}$ achieves pure fairness among all users when all users request uniform quality. We consider a four-user system, where each user receives 10 GOFs from one of the four video sequences, Foreman, Hall Monitor, Mother and daughter, and Silent, respectively. Figure 4(a) shows the received video quality of the first GOF in terms of mean squared error in every transmission interval. As we can see, all four users have similar video quality in each transmission interval and the received video quality is improved by receiving more quality layers till the last transmission interval. We also show the corresponding subcarrier assignment of the first GOF in each transmission interval in Figure 5(a). As the source coding rate of each user is allocated in different time and frequency slots according to the channel conditions and source characteristics, the diversities of frequency, time, 


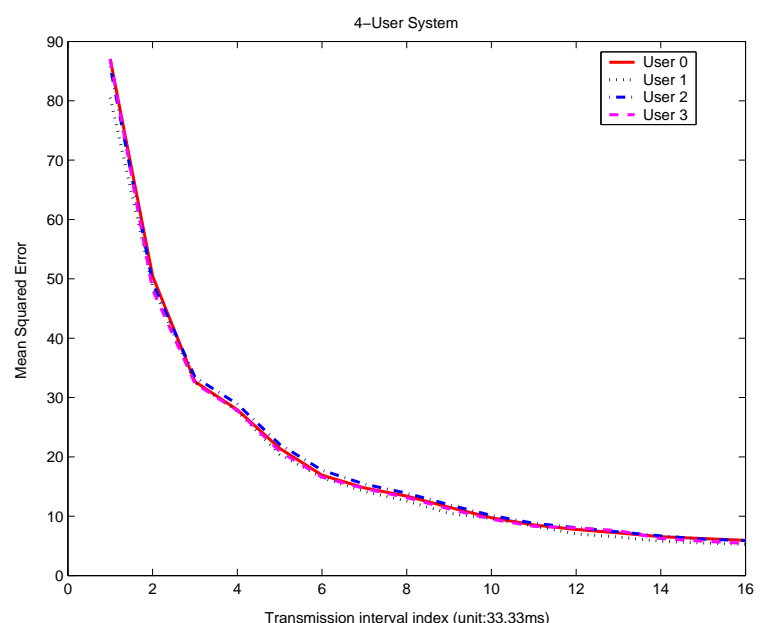

(a) $\mathrm{F}_{16} \mathrm{E}_{0}$ system

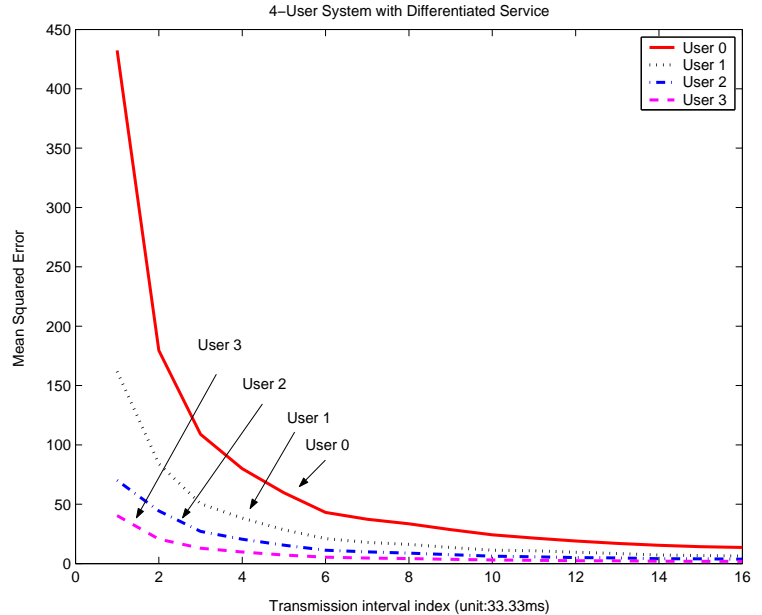

(b) $\mathrm{F}_{16} \mathrm{E}_{0}$ system with differentiated service

Fig. 4. Comparison for the $\mathrm{F}_{16} \mathrm{E}_{0}$ system providing uniform quality and differentiated service.

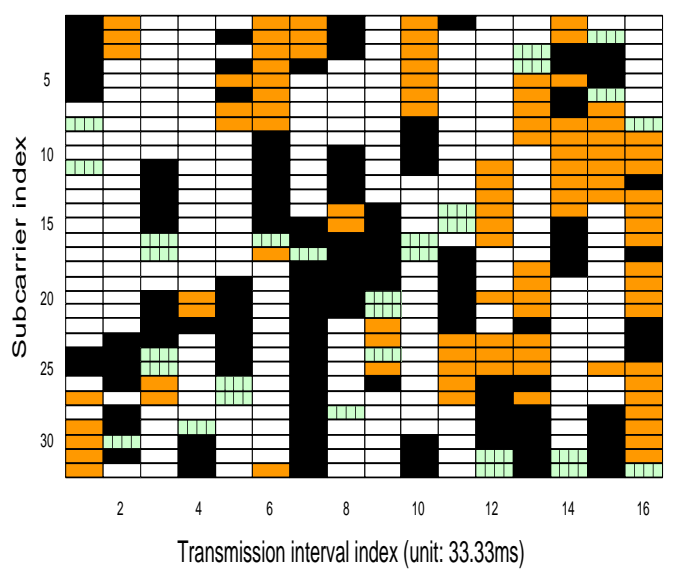

(a) $\mathrm{F}_{16} \mathrm{E}_{0}$ system

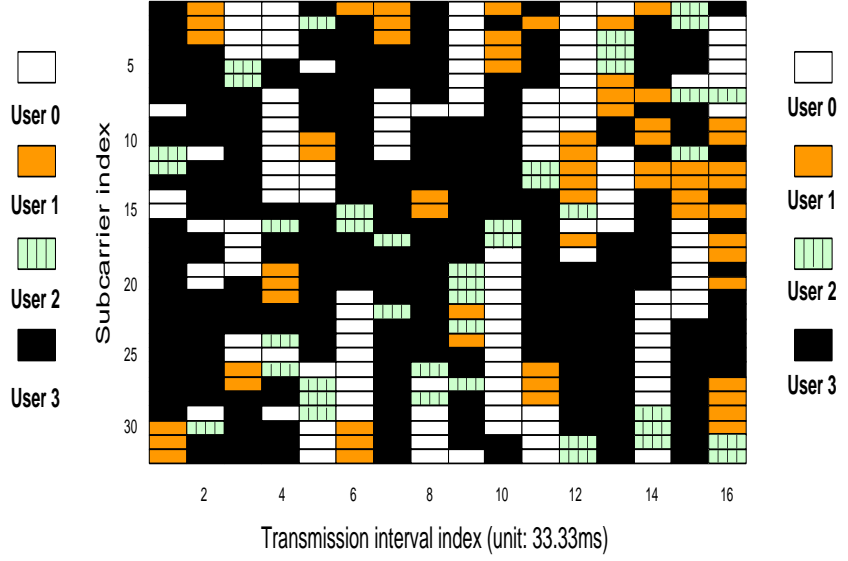

(b) $\mathrm{F}_{16} \mathrm{E}_{0}$ system with differentiated service

Fig. 5. Subcarrier assignment for the $\mathrm{F}_{16} \mathrm{E}_{0}$ system in each transmission interval (a) Uniform quality. The system assigns more subcarriers to user 0 at most intervals due to the required rate of video sequence 0 to achieve the same quality is higher than other sequences. (b) Differentiated service. The system assigns more subcarriers to user 3 due to the highest requested quality.

and multiuser are jointly exploited. Figure 6(a) shows the frame-by-frame PSNR along 10 GOFs for all users. The average PSNR along the received 160 frames for each user is $39.52,39.71,39.46,39.54 \mathrm{~dB}$, respectively. The deviation of users' received quality is small and within $0.25 \mathrm{~dB}$. Thus, the pure fairness algorithm, $\mathrm{F}_{16} \mathrm{E}_{0}$, can provide similar visual qualities among all users during the whole transmission time. 


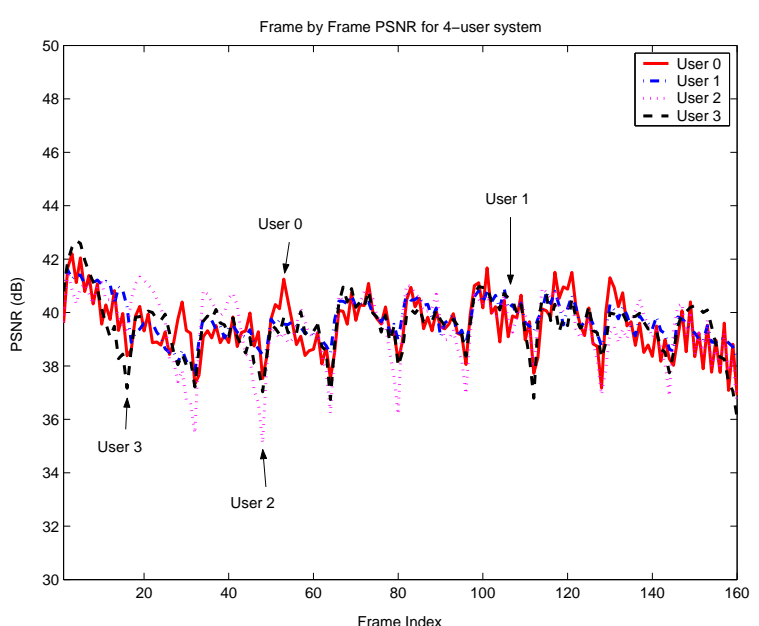

(a) $\mathrm{F}_{16} \mathrm{E}_{0}$ system

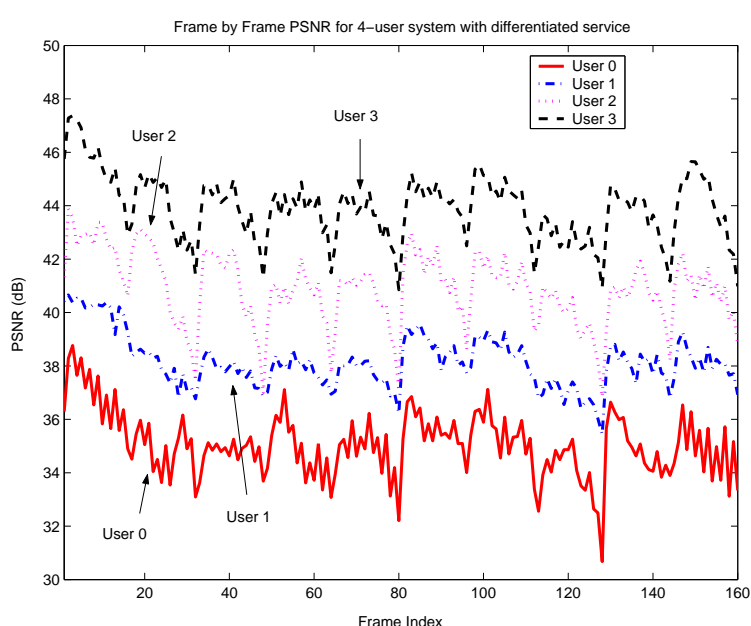

(b) $\mathrm{F}_{16} \mathrm{E}_{0}$ system with differentiated service

Fig. 6. Frame-by-frame PSNR for the $\mathrm{F}_{16} \mathrm{E}_{0}$ system with uniform quality and with differentiated service.

As we have mentioned that the proposed framework can provide differentiated service by appropriately setting the quality weighting factor $\left\{w_{k}\right\}$ in (10). We repeat the above experiment with a new set $\left\{w_{k}\right\}$ as $w_{0}=0.25, w_{1}=0.5, w_{2}=1$, and $w_{3}=2$. The PSNR difference between user $i$ and $i+1$ is expected to be $3 \mathrm{~dB}$. Figure 4(b) shows the mean squared error of the first GOF received by each user in every transmission interval. As we can see, the video qualities received by all users maintain the desired quality gap in every transmission interval. The differentiated service is achieved when we receive all quality layers. Figure 5(b) shows the corresponding subcarrier assignment of the first GOF in each transmission interval. Compared to Figure 5(a), User 3 occupies more subcarriers in the system with differentiated service than in the system with uniform quality. Figure 6(b) shows the frame-by-frame PSNR along 10 GOFs for all users. As we have expected, User 3 has the highest received video quality and User 0 has the lowest PSNR. The average PSNR received by each user is 35.06, 38.16, 40.91, 43.92dB, respectively. The PSNR differences between user $i$ and $i+1$ for $i=0,1,2$ are 3.10, 2.75, $3.01 \mathrm{~dB}$, respectively, which is close to the design goal of $3 \mathrm{~dB}$ differentiated service. 


\section{B. Performance Evaluation of the $F_{x} E_{y}$ Algorithm Family}

Next, we evaluate the proposed algorithm $\mathrm{F}_{x} \mathrm{E}_{y}$ with different values of $x$. Here we also compare the proposed algorithm with the TDM algorithm ${ }^{2}$. Instead of allowing subcarriers in a transmission interval to be allocated among multiple users, the TDM algorithm assigns all subcarriers in one transmission interval to only one user whose current end-to-end distortion is the largest. Thus, the multiuser and frequency diversity is not explored in this TDM algorithm.

We concatenate 15 classic CIF video sequences to form one testing video sequence of 4064 frames. The 15 sequences are 288-frame Akiyo, 144-frame Bus, 288-frame Coastguard, 288-frame Container, 240frame Flower, 288-frame Foreman, 288-frame Hall Monitor, 288-frame Highway, 288-frame Mobile, 288-frame Mother and daughter, 288-frame MPEG4 news, 288-frame Paris, 288-frame Silent, 256-frame Tempete, and 256-frame Waterfall. The video for the $k^{\text {th }}$ user is 160 frames long and from frame $256 \times k+1$ to frame $256 \times k+160$ of the testing sequence.

Two performance criteria are used to measure the proposed algorithm and TDM algorithm. We first calculate the average received video quality of all 160 frames for each user and denote it as $\mathrm{PSNR}_{k}$ for the $k^{t h}$ user. To measure the efficiency, we average the $\operatorname{PSNR}_{k}$ for all users, i.e.

$$
\operatorname{avePSNR}=\frac{1}{K} \sum_{k=0}^{K-1} \operatorname{PSNR}_{k} .
$$

The higher avePSNR is, the higher system efficiency of overall video quality we have. To measure the fairness, we take the standard deviation for each user's average received video quality, i.e.

$$
\operatorname{stdPSNR}=\sqrt{\frac{1}{K} \sum_{k=0}^{K-1}\left(\mathrm{PSNR}_{k}-{\operatorname{avePSNR})^{2}}^{2} .\right.}
$$

The lower stdPSNR is, the fairer quality each user receives. If stdPSNR is high, it implies some users receive video programs with high quality and the other users receive video programs with poor quality.

\footnotetext{
${ }^{2}$ The current IEEE 802.11 medium access control (MAC) protocol supports two kinds of access methods: distributed coordination function (DCF) and point coordination function (PCF). In both mechanisms, only one user occupies all the bandwidth at each time, which is similar to TDM technology.
} 


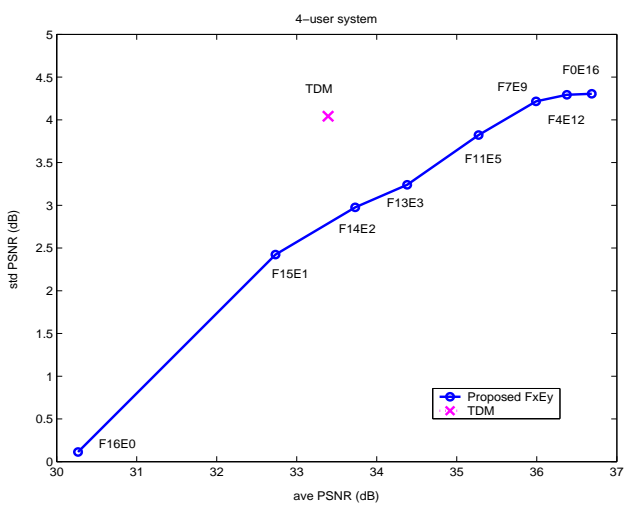

(a) 4-user system

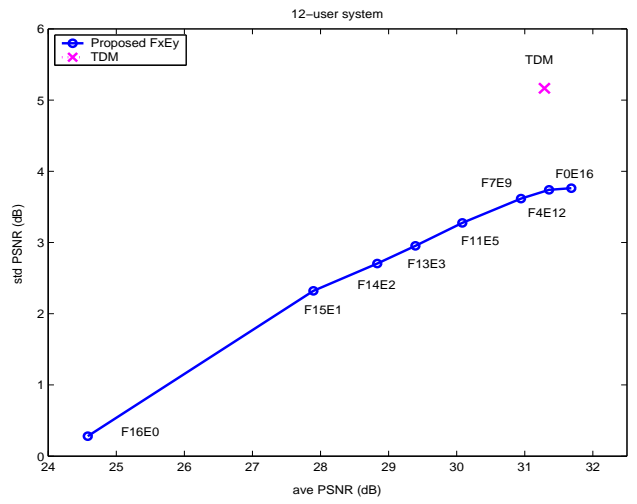

(c) 12-user system

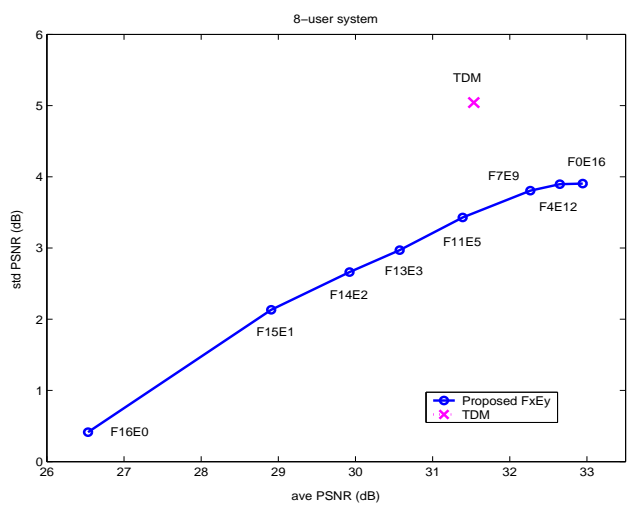

(b) 8-user system

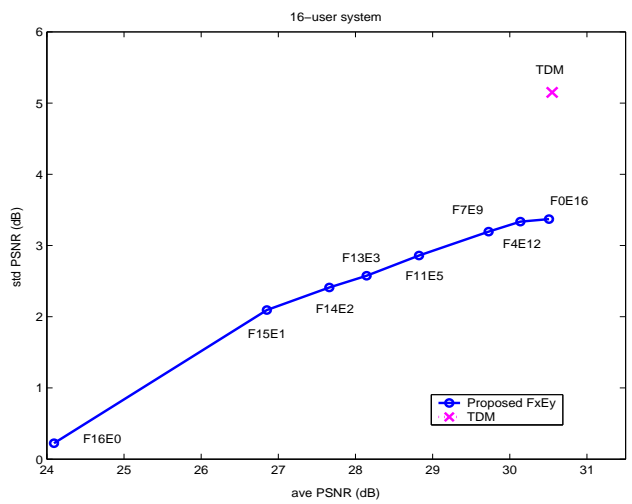

(d) 16-user system

Fig. 7. avePSNR and stdPSNR results of the $\mathrm{F}_{x} \mathrm{E}_{y}$ algorithm family and TDM algorithm.

Figure 7 shows the fairness and efficiency results for the proposed algorithms and TDM algorithm. We first compare the performances for eight settings of the $\mathrm{F}_{x} \mathrm{E}_{y}$ algorithm family, including $\mathrm{F}_{0} \mathrm{E}_{16}, \mathrm{~F}_{4} \mathrm{E}_{12}$, $\mathrm{F}_{7} \mathrm{E}_{9}, \mathrm{~F}_{11} \mathrm{E}_{5}, \mathrm{~F}_{13} \mathrm{E}_{3}, \mathrm{~F}_{14} \mathrm{E}_{2}, \mathrm{~F}_{15} \mathrm{E}_{1}$, and $\mathrm{F}_{16} \mathrm{E}_{0}$. We see that the pure fairness algorithm $\mathrm{F}_{16} \mathrm{E}_{0}$ achieves the lowest PSNR deviation among all algorithms but has the lowest average PSNR; and the pure efficiency algorithm $\mathrm{F}_{0} \mathrm{E}_{16}$ achieves the highest average PSNR but has the highest PSNR deviation. The tradeoff between avePSNR and stdPSNR can be adjusted by selecting different number of transmission intervals for fairness algorithm. As revealed from Figure 7, the $\mathrm{F}_{x} \mathrm{E}_{y}$ algorithm has higher average received video quality but higher quality deviation than the $\mathrm{F}_{x-1} \mathrm{E}_{y+1}$ algorithm. The second comparison included in Figure 7 is between the $\mathrm{F}_{x} \mathrm{E}_{y}$ algorithm family and the TDM algorithm. As shown in Figure 7, for achieving the same avePSNR, the proposed $\mathrm{F}_{x} \mathrm{E}_{y}$ algorithm family has about $1 \sim 1.8 \mathrm{~dB}$ lower deviation in PSNR than the TDM algorithm. In other words, the proposed algorithm provides fairer quality than 


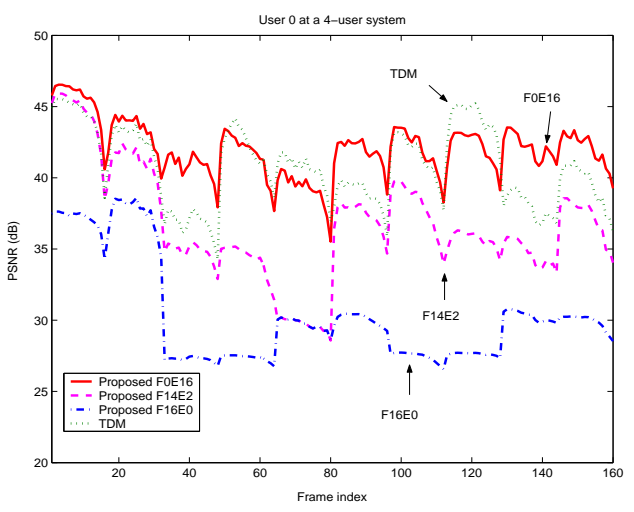

(a) User 0

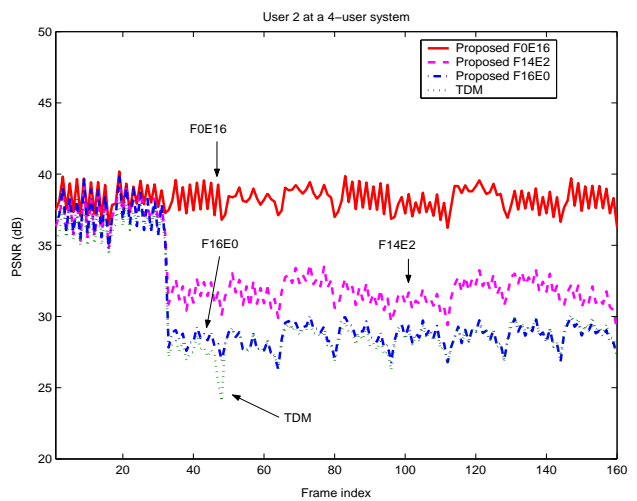

(c) User 2

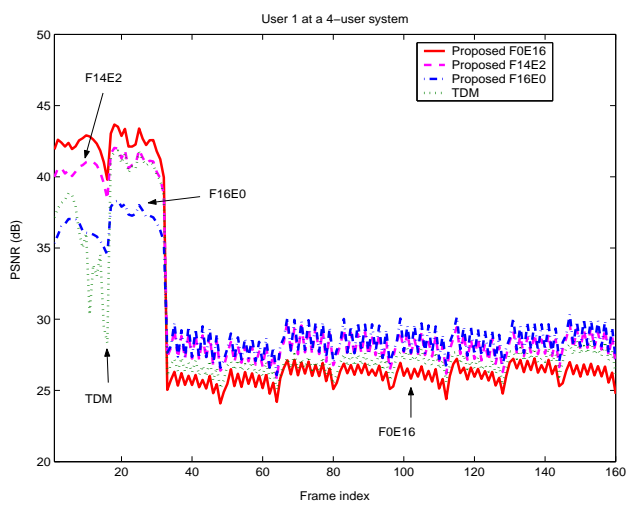

(b) User 1

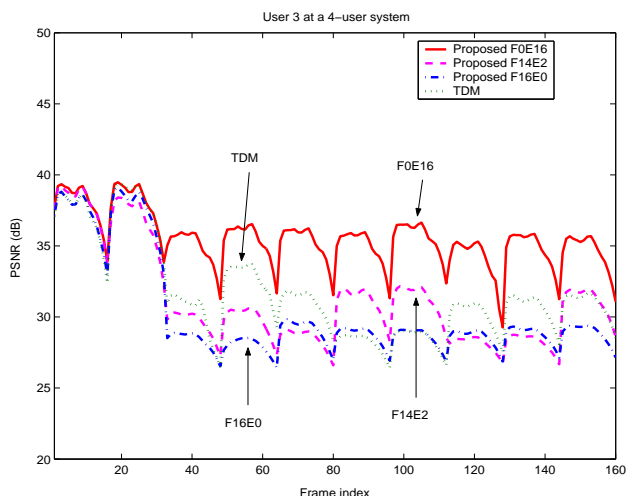

(d) User 3

Fig. 8. Frame-by-frame PSNR for different algorithms of a 4-user system.

the TDM algorithm. This is because the proposed scheme employs additional diversity in frequency and multiuser.

To evaluate the received video quality along the time axis, we show the frame-by-frame PSNR using TDM algorithm and the $\mathrm{F}_{x} \mathrm{E}_{y}$ algorithm family for each user in a four-user system in Figure 8. We choose three algorithms from $\mathrm{F}_{x} \mathrm{E}_{y}$ algorithm family, namely, the pure efficiency algorithm $\left(\mathrm{F}_{0} \mathrm{E}_{16}\right)$, the pure fairness algorithm $\left(\mathrm{F}_{16} \mathrm{E}_{0}\right)$, and one example of the partial fairness-efficiency algorithm $\left(\mathrm{F}_{14} \mathrm{E}_{2}\right)$. As we can see, the $\mathrm{F}_{0} \mathrm{E}_{16}$ algorithm has higher PSNR than $\mathrm{F}_{14} \mathrm{E}_{2}, \mathrm{~F}_{16} \mathrm{E}_{0}$ for all users except User 1. This is due to two factors: one is that the video content of User 1 requires higher rate to achieve the same video quality than the other users and the other reason is that the channel condition for User 1 is the worst among all users. Therefore, the $\mathrm{F}_{0} \mathrm{E}_{16}$ algorithm assigns more rates to the other users than User 1 to achieve higher average received video quality of all users. 


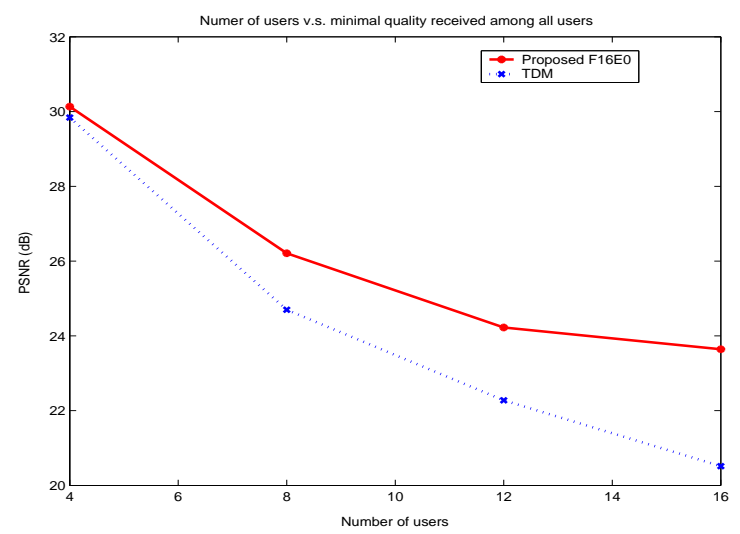

Fig. 9. Performance comparison for the worst quality received among all users using the proposed algorithm and TDM algorithm.

Figure 9 shows the average value of the worst received PSNR among all users from 10 different terminals' locations for different number of users in the system. We can see that the proposed algorithm, $\mathrm{F}_{16} \mathrm{E}_{0}$, can improve the minimal PSNR better than that of the TDM algorithm. There is about $0.5 \sim 3 \mathrm{~dB}$ gain for different number of users. The performance gap increases when the number of users increases owing to the multiuser diversity.

In Table VI, we show the performance gain that the unequal error protection scheme outperforms the equal error protection (EEP) for different numbers of users in the system using $\mathrm{F}_{0} \mathrm{E}_{16}$ algorithm. For the UEP strategy, the targeted BER of the first 15 transmission intervals is set to $10^{-6}$. The targeted BER of the last transmission interval is chosen from $\left\{10^{-5}, 10^{-6}\right\}$, depending on which BER setting achieving better expected distortion using (22). For the EEP, the targeted BER for all transmission intervals is $10^{-6}$. The video content and channel setting are the same as before. For each setting, we run the simulation 10,000 times. As revealed in Table VI, the UEP can improve the expected average PSNR per user only about $0.05 \sim 0.13 \mathrm{~dB}$.

This small improvement using the UEP is due to several reasons. First, although a system with higher BER has higher bit rate throughput, the distortion introduced by the channel becomes significant. Thus, the increased effective bandwidth is limited, which limits the reduction of video distortion. Second, the EWV codec has highly compression ratio at very low bit rate but its R-D curve becomes flatter at 
TABLE VI

PERFORMANCE GAIN OF USING UNEQUAL ERROR PROTECTION VERSUS USING EQUAL ERROR PROTECTION

\begin{tabular}{c||c|c|c|c}
\hline Number of users & 4 & 8 & 12 & 16 \\
\hline average PSNR gain per user $(\mathbf{d B})$ & 0.13 & 0.09 & 0.07 & 0.05 \\
\hline
\end{tabular}

higher bit rate due to the distortion-to-length slope sorting. So, for a system that has already received a large amount of video data at the last transmission interval, the improved distortion due to the increased effective bandwidth using the UEP is limited. Third, the joint multiple video coding has explored the video content complexities for all videos and the system has assigned more system resources to users who are in good channel conditions with simple video content complexity to achieve the highest system's efficiency. Thus, the extra bit rate budget benefited from the UEP will be distributed to users who are in bad channel conditions with complex video complexity, which can only improve a limited amount of overall distortion. Further, the selection of targeted BER is based on the expected distortion calculated from (22). If the targeted BER is selected as $10^{-6}$, the UEP is equivalent to the EEP and no performance gain can be obtained. We also observe that the more users the system has, the smaller performance improvement we have. It is because the increased bandwidth due to higher targeted BER is roughly a constant and is shared by all users. When the number of user increases, the increased bandwidth assigned to each user will reduce and the quality improvement will reduce.

\section{CONCLUSION}

In this paper, we have constructed a framework sending multiple scalable video programs over multiuser OFDM networks. By leveraging the frequency, time, and multiuser diversity of the OFDM system and the scalability of the 3-D embedded wavelet video codec, the proposed framework can allocate system resources to each video stream to achieve the desired video quality. Two service objectives are addressed: fairness and efficiency. For the fairness problem, we formulate the system to achieve fair quality among all users as a min-max problem. For the efficiency problem, we formulate the system to attain the lowest overall video distortion as a minimization problem. To satisfy the real-time requirement, two fast algorithms are proposed to solve the above two problems. 
The simulation results have demonstrated that the proposed fairness algorithm outperforms TDM algorithm by about $0.5 \sim 3 \mathrm{~dB}$ for the worst received video quality criterion. The proposed $\mathrm{F}_{x} \mathrm{E}_{y}$ algorithm family can achieve a desired tradeoff between fairness among users and overall system efficiency. At the same average video quality among all users, the proposed algorithm has about $1 \sim 1.8 \mathrm{~dB}$ lower PSNR deviation among all users than the TDM algorithm. So, the proposed algorithm can provide better system efficiency and stricter fairness. In addition, the proposed fairness algorithm can allow differentiated service by appropriately setting values for quality weighting factors. We also extend the proposed framework to incorporate unequal error protection. In summary, the proposed framework is a promising solution for broadband multiuser video communication.

\section{REFERENCES}

[1] H. Rohling and R. Grunheid, "Performance comparison of different multiple access schemes for the downlink of an OFDM communication system," Proc. IEEE Vehicular Technology Conference, pp.1365-1369, 1997.

[2] M. Wahlqvist, H. Olofsson, M. Ericson, C. Ostberg, and R. Larsson, "Capacity comparison of an OFDM based multiple access system using different dynamic resource allocation," Proc. IEEE Vehicular Technology Conference, vol.3, pp.16641668, May 1997.

[3] R. S. Cheng and S. Verdu, "Gaussian multiaccess channels with ISI: capacity region and multiuser water-filling," IEEE Transactions on Information Theory, vol.39, no.3, pp.773-785, May 1993.

[4] D. N. C. Tse and S. V. Hanly, "Multiaccess fading channels. I. Polymatroid structure, optimal resource allocation and throughput capacities," IEEE Transactions on Information Theory, vol.44, no.7, pp.2796-2815, Nov. 1998.

[5] S. Ulukus and R. D. Yates, "Iterative construction of optimum signature sequence sets in synchronous CDMA systems," IEEE Transactions on Information Theory, vol.47, no.5, pp.1989-1998, Jul. 2001.

[6] C. Y. Wong, R. S. Cheng, K. B. Lataief, and R. D. Murch, "Multiuser OFDM with adaptive subcarrier, bit, and power allocation,” IEEE Journal on Selected Areas in Communications, vol.17, no.10, pp.1747-1758, Oct. 1999.

[7] D. Kivanc, G. Li, and H. Liu, "Computationally efficient bandwidth allocation and power control for OFDMA," IEEE Transactions on Wireless Communications, vol.2, no.6, pp.1150-1158, Nov. 2003.

[8] P. S. Chow, J. M. Cioffi, and J. A. C. Bingham, "A practical discrete multitone transceiver loading algorithm for data transmission over spectrally shaped channels," IEEE Transactions on Communications, vol.43, no.2/3/4, pp.773-775, Feb./Mar./Apr. 1995.

[9] Z. Han, Z. Ji, and K. J. R. Liu, "Low-complexity OFDMA channel allocation with Nash bargaining solution fairness," IEEE Globel Telecommunication Conf., 2004.

[10] Z. Han, Z. Ji, and K. J. Ray Liu, "Power minimization for multi-cell OFDM networks using distributed non-cooperative game approach", IEEE Globel Telecommunication Conf., 2004.

[11] D. Taubman and A. Zakhor, "Multirate 3-D subband coding of video," IEEE Transactions on Image Processing, vol.3, no.5, pp.572-588, Sep. 1994. 
[12] J.-R. Ohm, "Three-dimensional subband coding with motion compensation," IEEE Transactions on Image Processing, vol.3, no.5, pp.559-571, Sep. 1994.

[13] C. I. Podilchuk, N. S. Jayant, and N. Farvardin, “Three-dimensional subband coding of video," IEEE Transactions on Image Processing, vol.4, no.2, pp.125-139, Feb. 1995.

[14] S.-J. Choi and J. W. Woods, "Motion-compensated 3-D subband coding of video," IEEE Transactions on Image Processing, vol.8, no.2, pp.155-167, Feb. 1999.

[15] B.-J. Kim, Z. Xiong, and W. A. Pearlman, "Low bit-rate scalable video coding with 3-D set partitioning in hierarchical trees (3-D SPIHT)," IEEE Transactions on Circuits and Systems for Video Technology, vol.10, no.8, pp.1374-1387, Dec. 2000.

[16] J. Hua, Z. Xiong, and X. Wu, "High-performance 3-D embedded wavelet video (EWV) coding," IEEE Fourth Workshop on Multimedai Signal Processing, 2001.

[17] D. S. Taubman, "High performance scalable image compression with EBCOT," IEEE Transactions on Image Processing, vol.9, no.7, pp.1158-1170, Jul. 2000.

[18] P. J. Cherriman, T. Keller, and L. Hanzo, "Orthogonal frequency-division multiplex transmission of H.263 encoded video over highly frequency-selective wireless networks," IEEE Transactions on Circuits and Systems for Video Technology, vol.9, no.5, pp.701-712, Aug. 1999.

[19] B. Girod and N. Farber, Wireless video, in Compressed video over networks, M-T. Sun and A.R. Reibman, Eds. New York, Marcel Dekker, Inc, 2001.

[20] R. Yan, F. Wu, S. Li, R. Tao, and Y. Zhang, "Error resilience methods for FGS enhancement bitstream," The First IEEE Pacific-Rim Conference on Multimedia (PCM), pp.148-151, Dec. 2000.

[21] J. Xu, Q. Zhang, W. Zhu, X.-G. Xia, and Y.-Q. Zhang, "Optimal joint source-channel bit allocation for MPEG-4 fine granularity scalable video over OFDM system," IEEE International Symposium on Circuits and Systems vol.2, May 2003.

[22] H. Zheng and K. J. R. Liu, "The subband modulation: a joint power and rate allocation framework for subband image and video transmission," IEEE Transactions on Circuits and Systems for Video Technology, vol 9, no 5, pp.823-838, Aug. 1999.

[23] J. Song and K. J. R. Liu, "An integrated source and channel rate allocation scheme for robust video coding and transmission over wireless channels," EURASIP Journal on Applied Signal Processing, vol.2004, no.2, pp.304-316, Feb. 2004.

[24] H. Zheng and K. J. R. Liu, "Robust image and video transmission over spectrally shaped channels using multicarrier modulation,” IEEE Transactions on Multimedia, vol.1, no.1, pp.88-103, Mar. 1999.

[25] J. Song and K. J. R. Liu, "Robust progressive image transmission over OFDM systems using space-time block code," IEEE Transactions on Multimedia, vol.4, no.3, pp.394-406, Sep. 2002.

[26] Z. Ji, Q. Zhang, W. Zhu, J. Lu, and Y.-Q. Zhang, "Video broadcasting over MIMO-OFDM systems," IEEE International Symposium on Circuits and Systems, vol.2, pp.844-847, May 2003.

[27] H. Cai, B. Zeng, G. Shen, Z. Xiong, and S. Li, "Error-resilient unequal error protection of fine granularity scalable video bitstreams," EURASIP Journal on Applied Signal Processing, vol.2006, Article ID 45412, 2006.

[28] L. Wang and A. Vincent, "Bit allocation and constraints for joint coding of multiple video programs," IEEE Transactions on Circuits and Systems for Video Technology, vol.9, no.6, pp.949-959, Sep. 1999.

[29] X. M. Zhang, A. Vetro, Y. Q. Shi, and H. Sun, “Constant quality constrained rate allocation for FGS-coded videos,” IEEE Transactions on Circuits and Systems for Video Technology, vol.13, no.2, pp. 121-130, Feb. 2003. 
[30] G.-M. Su and M. Wu, "Efficient bandwidth resource allocation for low-delay multiuser MPEG-4 video transmission", IEEE Trans. on Circuits and Systems for Video Technology, vol. 15, no.9, pp.1124-1137, Sep. 2005.

[31] Z. Han, G.-M. Su, A. Kwasinski, M. Wu, and K. J. R. Liu, "Multiuser distortion management of layered video over resource limited downlink MC-CDMA”, accepted by IEEE Trans. on Wireless Communication, Jan. 2006.

[32] G.-M. Su, Z. Han, M. Wu, and K. J. R. Liu, “ Dynamic distortion control for 3-D embedded wavelet video over multi-user OFDM networks,” IEEE Globel Telecommunication Conf., vol.2, pp.650-654, 2004.

[33] J. Gross, J. Klaue, H. Karl, and A. Wolisz, "Subcarrier allocation for variable bit rate video streams in wireless OFDM systems," IEEE Vehicular Technology Conference, Fall. 2003

[34] C. E. Koksal and H. Kassab and H. Balakrishnan, "An Analysis of Short-Term Fairness in Wireless Media Access Protocols," ACM Sigmetrics 2000, Jun. 2000.

[35] M. H. Ahmed, H. Yanikomeroglu, and S. Mahmoud, "Fairness enhancement of link adaptation techniques in wireless networks," IEEE Vehicular Technology Conference, vol.4, pp.1554-1557, Fall 2003.

[36] S. Martello and P. Toth, "Knapsack Problems: Algorithms and Computer Implementations," Wiley, West Sussex, England, 1990.

[37] A. Albanese, J. Blomer, J. Edmonds, M. Luby, and M. Sudan, "Priority encoding transmission," IEEE Transactions on Information Theory, vol.42, no.6, pp.1737-1744, Nov. 1996.

[38] A. E. Mohr, E. A. Riskin, and R. E. Ladner, "Unequal loss protection: graceful degradation of image quality over packet erasure channels through forward error correction," IEEE Journal on Selected Areas in Communications, vol.18, no.6, pp.819828, Jun. 2000.

[39] P. G. Sherwood and K. Zeger, "Error protection for progressive image transmission over memoryless and fading channels," IEEE Transactions on Communications, vol.46, no.12, pp.1555 1559, Dec. 1998.

[40] T. S. Rappaport, Wireless Communications: principles and practice: 2nd edition, Prentice Hall, 2002.

\begin{tabular}{|c|}
\hline \\
PLACE \\
PHOTO \\
HERE \\
\hline
\end{tabular}

Guan-Ming Su (S’04) received the B.S.E. degree from National Taiwan University, Taipei, Taiwan, in 1996 and the M.S. degree from University of Maryland, College Park, in 2001, both in electrical engineering. He is currently working toward the Ph.D. degree in the Department of Electrical and Computer Engineering at the University of Maryland, College Park.

He is with ESS Technology, Inc., Fremont, CA. His research interests are multimedia communications and multimedia signal processing. 
Zhu Han (S'01-M'04) received the B.S. degree in electronic engineering from Tsinghua University, in

PLACE

PHOTO

HERE 1997, and the M.S. and Ph.D. degrees in electrical engineering from the University of Maryland, College Park, in 1997 and 2003, respectively.

From 1997 to 2000, he was a Graduate Research Assistant at the University of Maryland. From 2000 to 2002, he was an Engineer in the R\&D Group of ACTERNA, Maryland. From 2003 to 2006, he was a Research Associate at the University of Maryland. During Summer 2006, he was a visitor in Princeton University. Currently $\mathrm{He}$ is an assistant Professor in Electrical and Computer Engineering Department at Boise State University, Idaho, USA. His research interests include wireless resource allocation and management, wireless communications and networking, game theory, and wireless multimedia.

Dr. Han is a member of the Technical Programming Committee for the IEEE International Conference on Communications of 2004, 2005, and 2007, the IEEE Vehicular Technology Conference, Spring 2004, the IEEE Consumer Communications and Networking Conference 2005, 2006, and 2007, the IEEE Wireless Communications and Networking Conference 2005,2006 and 2007, and the IEEE Globe Communication Conference 2005 and 2006, as well as Session Chair of the IEEE Wireless Communications and Networking Conference 2004, 2005, 2006 and the IEEE Globe Communication Conference 2005.

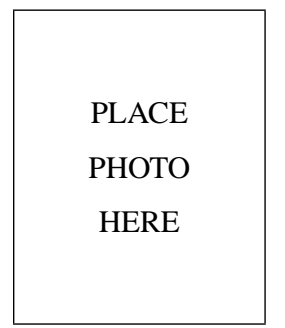

Min Wu (S'95-M'01) received the B.E. degree in electrical engineering and the B.A. degree in economics (both with the highest honors) from Tsinghua University, Beijing, China, in 1996, and the Ph.D. degree in electrical engineering from Princeton University in 2001.

Since 2001, she has been on the faculty of the Department of Electrical and Computer Engineering and the Institute of Advanced Computer Studies at the University of Maryland, College Park, where she is currently an Associate Professor. Previously she was with the NEC Research Institute and Panasonic Laboratories. She co-authored two books, Multimedia Data Hiding (Springer-Verlag, 2003) and Multimedia Fingerprinting Forensics for Traitor Tracing (EURASIP/Hindawi, 2005), and holds five U.S. patents. Her research interests include information security and forensics, multimedia signal processing, and multimedia communications.

Dr. Wu is an Associate Editor of the IEEE Signal Processing Letters and a member of the IEEE Technical Committee on Multimedia Systems and Applications. She received an NSF CAREER award in 2002, a University of Maryland George Corcoran Education Award in 2003, an MIT Technology Review's TR100 Young Innovator Award in 2004, and an ONR Young Investigator Award in 2005. She is a co-recipient of the 2004 EURASIP Best Paper Award and the 2005 IEEE Signal Processing Society Best Paper Award. 
K. J. Ray Liu (F’03) is Professor and Associate Chair, Graduate Studies and Research, of Electrical and Computer Engineering Department, University of Maryland, College Park. His research contributions

PLACE

PHOTO encompass broad aspects of wireless communications and networking, information forensics and security, multimedia communications and signal processing, bioinformatics and biomedical imaging, and signal processing algorithms and architectures.

Dr. Liu is the recipient of numerous honors and awards including best paper awards from IEEE Signal Processing Society (twice), IEEE Vehicular Technology Society, and EURASIP; IEEE Signal Processing Society Distinguished Lecturer, EURASIP Meritorious Service Award, and National Science Foundation Young Investigator Award. He also received various teaching and research awards from University of Maryland including Poole and Kent Company Senior Faculty Teaching Award and Invention of the Year Award.

Dr. Liu is Vice President - Publications and on the Board of Governor of IEEE Signal Processing Society. He was the Editor-in-Chief of IEEE Signal Processing Magazine and the founding Editor-in-Chief of EURASIP Journal on Applied Signal Processing. 